\title{
Cramér-type Moderate Deviation Theorems for Nonnormal Approximation
}

\author{
Qi-Man Shao ${ }^{1,2 *}$, Mengchen Zhang ${ }^{3}$ and \\ Zhuo-Song Zhang ${ }^{2,4 \dagger}$ \\ Department of Statistics and Data Scinece \\ Southern University of Science and Technology \\ Shenzhen, Guangdong, P.R. China \\ and \\ Department of Statistics \\ The Chinese University of Hong Kong \\ Shatin, N.T., Hong Kong \\ e-mail: qmshao@cuhk.edu.hk \\ Department of Mathematics \\ Hong Kong University of Science and Technology \\ Clear Water Bay \\ Kowloon, Hong Kong \\ e-mail: mzhangag@connect.ust.hk \\ Department of Statistics \\ The Chinese University of Hong Kong \\ Shatin, N.T., Hong Kong \\ and \\ Department of Statistics and Applied Probability \\ National University of Singapore \\ Singapore 117546 \\ e-mail: zszhang.stat@gmail.com
}

\begin{abstract}
A Cramér-type moderate deviation theorem quantifies the relative error of the tail probability approximation. It provides a criterion whether the limiting tail probability can be used to estimate the tail probability under study. Chen, Fang and Shao (2013) obtained a general Cramér-
\end{abstract}

\footnotetext{
* Research partially supported by Hong Kong RGC GRF 14302515 and 14304917

${ }^{\dagger}$ Corresponding author. Research partially supported by Singapore Ministry of Education Academic Research Fund MOE 2018-T2-076 


\begin{abstract}
type moderate result using Stein's method when the limiting was a normal distribution. In this paper, Cramér-type moderate deviation theorems are established for nonnormal approximation under a general Stein identity, which is satisfied via the exchangeable pair approach and Stein's coupling. In particular, a Cramér-type moderate deviation theorem is obtained for the general Curie-Weiss model and the imitative monomer-dimer meanfield model.
\end{abstract}

MSC2020 subject classifications: Primary 60F10; secondary 60F05.

Keywords and phrases: Moderate deviation, Nonnormal approximation, Stein's method, Curie-Weiss model, Imitative monomer-dimer mean-field model.

\title{
1. Introduction
}

Consider a sequence of random variables $W_{n}$. One often needs to calculate the tail probability of $W_{n}$ such as $\mathrm{P}\left(W_{n} \geq x_{n}\right)$. Since the exact distribution of $W_{n}$ is hardly known, it is common to use the limiting distribution, that is, assuming that $W_{n}$ converges to $Y$ in distribution, $\mathrm{P}\left(Y \geq x_{n}\right)$ is used to estimate $\mathrm{P}\left(W_{n} \geq x_{n}\right)$. The Cramér-type moderate deviation seeks the largest possible $a_{n}$ so that

$$
\frac{\mathrm{P}\left(W_{n} \geq x\right)}{\mathrm{P}(Y \geq x)}=1+\text { error } \rightarrow 1
$$

holds for $0 \leq x \leq a_{n}$. This quantifies the relative error of the distribution approximation and provides a criterion whether the limiting tail probability can be used to estimate the tail probability. When $Y$ is the normal random variable and $W_{n}$ is the standardized sum of the independent random variables, the Cramértype moderate deviation is well understood. In particular, for independent and identically distributed random variables $X_{1}, \ldots, X_{n}$ with $\mathrm{E} X_{i}=0, \mathrm{E} X_{i}^{2}=1$ and $\mathrm{E} e^{t_{0}} \sqrt{\left|X_{1}\right|}<\infty, t_{0}>0$, it holds that

$$
\frac{\mathrm{P}\left(W_{n} \geq x\right)}{1-\Phi(x)}=1+O(1)\left(1+x^{3}\right) / \sqrt{n}
$$

for $0 \leq x \leq n^{1 / 6}$, where $W_{n}=\left(X_{1}+\cdots+X_{n}\right) / \sqrt{n}$. The finite-momentgenerating function of $\left|X_{1}\right|^{1 / 2}$ is necessary, and both the range $0 \leq x \leq n^{1 / 6}$ and the order of the error term $\left(1+x^{3}\right) / \sqrt{n}$ are optimal. We refer to Linnik [20] and Petrov [23, p. 251] for details.

Considering general dependent random variables whose dependence is defined in terms of a Stein identity, Chen, Fang and Shao [12] obtained a general 
Cramér-type moderate deviation result for normal approximation using Stein's method. Stein's method, introduced by Stein [28], is a completely different approach to distribution approximation than the classical Fourier transform. It works not only for independent random variables but also for dependent random variables. It can also provide accuracy of the distribution approximation. Extensive applications of Stein's method to obtain Berry-Esseen-type bounds can be found in, for example, Diaconis [16], Stein [29], Barbour [3], Goldstein and Reinert [19], Chen and Shao [10, 11], Chatterjee [6], Nourdin and Peccati [21] and Shao and Zhang [26]. We refer to Chen, Goldstein and Shao [13], Nourdin and Peccati [22] and Chatterjee [7] for comprehensive coverage of the method's fundamentals and applications. In addition to the normal approximation, Chatterjee and Shao [9] obtained a general nonnormal approximation via the exchangeable pair approach and the corresponding Berry-Esseen-type bounds. We also refer to Shao and Zhang [25] for a more general result.

The main purpose of this paper is to obtain a Cramér-type moderate deviation theorem for nonnormal approximation. Our main tool is based on Stein's method, combined with some techniques in Chatterjee and Shao [9] and Chen, Fang and Shao [12]. The paper is organized as follows. Section 2 presents a Cramér-type moderate deviation theorem under a general Stein identity setting, which recovers the result of Chen, Fang and Shao [12] as a special case. In Section 3, the result is applied to two examples: the general Curie-Weiss model and imitative monomer-dimer models. The proofs of the main results in Section 2 are given in Sections 4 and the proofs of theorems in Section 3 are postponed to Section 5 .

\section{Main Results}

Let $W:=W_{n}$ be the random variable of interest. Following the setting in Chatterjee and Shao [9] and Chen, Fang and Shao [12], we assume that there exists a constant $\delta$, a nonnegative random function $\hat{K}(t)$, a function $g$ and a random variable $R(W)$ such that

$$
\mathrm{E}(f(W) g(W))=\mathrm{E}\left(\int_{|t| \leq \delta} f^{\prime}(W+t) \hat{K}(t) d t\right)+\mathrm{E}(f(W) R(W))
$$


Q.M. Shao, M.C. Zhang and Z.S. Zhang/Moderate deviation for nonnormal approximationt

for all absolutely continuous functions $f$ for which the expectation of either side exists. Let

$$
\hat{K}_{1}=\int_{|t| \leq \delta} \hat{K}(t) d t
$$

and

$$
G(y)=\int_{0}^{y} g(t) d t
$$

Let $Y$ be a random variable with the probability density function

$$
p(y)=c_{1} e^{-G(y)}, \quad y \in \mathbb{R},
$$

where $c_{1}$ is a normalizing constant.

In this section, we present a Cramér-type moderate deviation theorem for general distribution approximation under Stein's identity in general and under an exchangeable pair and Stein's couplings in particular.

Before presenting the main theorem, we first give some of the conditions of $g$.

Assume that

(A1) The function $g$ is nondecreasing and $g(0)=0$.

(A2) For $y \neq 0, y g(y)>0$.

(A3) There exists a positive constant $c_{2}$ such that for $x, y \in \mathbb{R}$,

$$
|g(x+y)| \leq c_{2}(|g(x)|+|g(y)|+1) .
$$

(A4) There exists $c_{3} \geq 1$ such that for $y \in \mathbb{R}$,

$$
\left|g^{\prime}(y)\right| \leq c_{3}\left(\frac{1+|g(y)|}{1+|y|}\right) .
$$

A large class of functions satisfy conditions (A1)-(A4). A typical example is $g(y)=\operatorname{sgn}(y)|y|^{p}, p \geq 1$.

We are now ready to present our main theorem.

Theorem 2.1. Let $W$ be a random variable of interest satisfying (2.1). Assume that conditions (A1)-(A4) are satisfied. Additionally, assume that there exist $\tau_{1}>0, \tau_{2}>0, \delta_{1}>0$ and $\delta_{2} \geq 0$ such that

$$
\begin{aligned}
\left|\mathrm{E}\left(\hat{K}_{1} \mid W\right)-1\right| & \leq \delta_{1}\left(|g(W)|^{\tau_{1}}+1\right), \\
|R(W)| & \leq \delta_{2}\left(|g(W)|^{\tau_{2}}+1\right) .
\end{aligned}
$$


Q.M. Shao, M.C. Zhang and Z.S. Zhang/Moderate deviation for nonnormal approximations

In addition, there exist constants $d_{0} \geq 1, d_{1}>0$ and $0 \leq \alpha<1$ such that

$$
\begin{aligned}
\mathrm{E}\left(\hat{K}_{1} \mid W\right) & \leq d_{0}, \\
\delta|g(W)| & \leq d_{1}, \\
|R(W)| & \leq \alpha(|g(W)|+1) .
\end{aligned}
$$

Then, we have

$$
\begin{aligned}
\frac{\mathrm{P}(W>z)}{\mathrm{P}(Y>z)}=1+O(1)( & \delta\left(1+z g^{2}(z)\right) \\
& \left.+\delta_{1}\left(1+z g^{\tau_{1}+1}(z)\right)+\delta_{2}\left(1+z g^{\tau_{2}}(z)\right)\right)
\end{aligned}
$$

for $z \geq 0$ satisfying $\delta z g^{2}(z)+\delta_{1} z g^{\tau_{1}+1}(z)+\delta_{2} z g^{\tau_{2}}(z) \leq 1$, where $O(1)$ is bounded by a finite constant depending only on $d_{0}, d_{1}, c_{1}, c_{2}, c_{3}, \tau_{1}, \tau_{2}, \alpha$ and $\max (g(1),|g(-1)|)$.

The condition (2.1) is called a general Stein identity, see Chen, Goldstein and Shao [13, Chapter 2]. We use the exchangeable pair approach and Stein's coupling to construct $\hat{K}(t)$ and $R(W)$ as follows.

Let $\left(W, W^{\prime}\right)$ be an exchangeable pair, that is, $\left(W, W^{\prime}\right)$ has the same joint distribution as $\left(W^{\prime}, W\right)$. Let $\Delta=W-W^{\prime}$. Assume that

$$
\mathrm{E}(\Delta \mid W)=\lambda(g(W)-R(W))
$$

where $0<\lambda<1$. Assume that $|\Delta| \leq \delta$ for some constant $\delta>0$. It is known (see, e.g., Chatterjee and Shao [9]) that (2.1) is satisfied with

$$
\hat{K}(t)=\frac{1}{2 \lambda} \Delta(I(-\Delta \leq t \leq 0)-I(0<t \leq \Delta)) .
$$

Clearly, we have

$$
\hat{K}_{1}=\frac{1}{2 \lambda} \Delta^{2}
$$

For exchangeable pairs, we have the following corollary.

Corollary 2.1. For $\left(W, W^{\prime}\right)$ an exchangeable pair satisfying (2.13), assume that $g(W), \hat{K}_{1}$ and $R(W)$ satisfy the conditions (A1)-(A4) and (2.7)-(2.11) stated in Theorem 2.1; then, (2.12) holds.

Stein's coupling introduced by Chen and Röllin [14] is another way to construct the general Stein identity.

imsart-generic ver. 2020/08/06 file: AAP1589-final.tex date: April 28, 2021 
Q.M. Shao, M.C. Zhang and Z.S. Zhang/Moderate deviation for nonnormal approximation

A triple $\left(W, W^{\prime}, T\right)$ is called a $g$-Stein's coupling if there is a function $g$ such that

$$
\mathrm{E}\left(T f\left(W^{\prime}\right)-T f(W)\right)=\mathrm{E}(f(W) g(W))
$$

for all absolutely continuous function $f$, such that the expectations on both sides exist. Assume that $\left|W^{\prime}-W\right| \leq \delta$. Then, by Chen and Röllin [14], we have

$$
\mathrm{E}(f(W) g(W))=\mathrm{E}\left(\int_{|t| \leq \delta} f^{\prime}(W+t) \hat{K}(t) d t\right)
$$

where

$$
\hat{K}(t)=T\left(I\left(0 \leq t \leq W^{\prime}-W\right)-I\left(W^{\prime}-W \leq t<0\right)\right) .
$$

It is easy to see that $\hat{K}_{1}=T\left(W^{\prime}-W\right)$.

The following corollary presents a moderate deviation result for Stein's coupling.

Corollary 2.2. Let $\left(W, W^{\prime}, T\right)$ be a g-Stein's coupling satisfying (2.14) and (2.15) and let $\hat{K}$ be defined as in (2.16) and assume that $\hat{K}(t) \geq 0$ for $|t| \leq \delta$. Let $g(W)$ and $\hat{K}_{1}:=T\left(W^{\prime}-W\right)$ satisfy the conditions (A1)-(A4) and (2.7), (2.9) and (2.10) stated in Theorem 2.1, then (2.12) holds with $\delta_{2}=0$.

Remark 2.1. For $s \geq 0$, let

$$
\zeta(w, s)= \begin{cases}e^{G(w)-G(w-s)}, & w>s \\ e^{G(w)}, & 0 \leq w \leq s \\ 1, & w<0\end{cases}
$$

Condition (2.7) can be replaced by

$$
\left|\mathrm{E}\left(\hat{K}_{1} \mid W\right)-1\right| \leq K_{2}+\delta_{1}\left(|g(W)|^{\tau_{1}}+1\right),
$$

where $K_{2} \geq 0$ is a random variable satisfying

$$
\mathrm{E} K_{2} \zeta(W, s) \leq \delta_{1}\left(1+g^{\tau_{1}}(s)\right) \mathrm{E} \zeta(W, s)
$$

Remark 2.2. Condition (2.11) may not be satisfied when $|W|$ is large in some applications. Following the proof of Theorem 2.1, when (2.11) is replaced by the following condition, there exist $0 \leq \alpha<1, d_{2} \geq 0, d_{3}>0$ and $\kappa>0$ such that

$$
|R(W)| \leq \alpha(|g(W)|+1)+d_{2} I(|W|>\kappa),
$$


Q.M. Shao, M.C. Zhang and Z.S. Zhang/Moderate deviation for nonnormal approximation

and

$$
d_{2} \mathrm{P}(|W|>\kappa) \leq d_{3} e^{-2 s_{0} d_{1}^{-1} \delta^{-1}},
$$

where $d_{1}$ is bounded in (2.10) and $s_{0}=\max \left\{s: \delta s g^{2}(s) \leq 1\right\}$, Theorem 2.1 and Corollaries 2.1 and 2.2 remain valid with $O(1)$ bounded by a finite constant depending only on $d_{0}, d_{1}, d_{2}, d_{3}, c_{1}, c_{2}, c_{3}, \tau_{1}, \tau_{2}, \alpha$ and $\max (g(1),|g(-1)|)$.

\section{Applications}

In this section, we apply the main results to the general Curie-Weiss model at the critical temperature and the imitative monomer-dimer model.

\subsection{General Curie-Weiss model at the critical temperature}

Let $\xi$ be a random variable with probability measure $\rho$ which is symmetric on $\mathbb{R}$. Assume that

$$
\mathrm{E} \xi^{2}=1, \quad \mathrm{E} \exp \left(\beta \xi^{2} / 2\right)<\infty \quad \text { for } \quad \beta \geq 0
$$

The general Curie-Weiss model $\mathrm{CW}(\rho)$ at inverse temperature $\beta$ is defined as the array of spin random variables $\mathbf{X}=\left(X_{1}, X_{2}, \ldots, X_{n}\right)$ with joint distribution

$$
\mathrm{dP}_{n}(\mathbf{x})=Z_{n}^{-1} \exp \left(\frac{\beta}{2 n}\left(x_{1}+x_{2}+\cdots+x_{n}\right)^{2}\right) \prod_{i=1}^{n} \mathrm{~d} \rho\left(x_{i}\right)
$$

for $\mathbf{x}=\left(x_{1}, x_{2}, \ldots, x_{n}\right) \in \mathbb{R}^{n}$ where

$$
Z_{n}=\int \exp \left(\frac{\beta}{2 n}\left(x_{1}+x_{2}+\cdots+x_{n}\right)^{2}\right) \prod_{i=1}^{n} \mathrm{~d} \rho\left(x_{i}\right)
$$

is the normalizing constant.

The magnetization $m(\mathbf{x})$ is defined by

$$
m(\mathbf{x})=\frac{1}{n} \sum_{i=1}^{n} x_{i} .
$$

Following the setting of Chatterjee and Dey [8], we assume that the measure $\rho$ satisfies the following conditions:

(B1) $\rho$ has compact support, that is, $\rho([-L, L])=1$ for some $L<\infty$. 
(B2) Let

$$
h(s):=\frac{s^{2}}{2}-\log \int \exp (s x) \mathrm{d} \rho(x) .
$$

The equation $h^{\prime}(s)=0$ has a unique root at $s=0$.

(B3) Let $k \geq 2$ be such that $h^{(i)}(0)=0$ for $0 \leq i \leq 2 k-1$ and $h^{(2 k)}(0)>0$.

Specially, for the simple Curie-Weiss model, where $\rho=\frac{1}{2} \boldsymbol{\delta}_{1}+\frac{1}{2} \boldsymbol{\delta}_{-1}$ and $\boldsymbol{\delta}$ is the Dirac measure, conditions (B1)-(B3) are satisfied with $L=1$ and $k=2$. For $0<\beta<1, n^{1 / 2} m(\mathbf{X})$ converges weakly to a Gaussian distribution, see Ellis and Newman [17]. Also, Chen, Fang and Shao [12] obtained the Cramér-type moderate deviation for this normal approximation. When $\beta=1$, Simon and Griffiths [27] proved that the law of $n^{1 / 4} m(\mathbf{X})$ converges to $\mathcal{W}(4,12)$ as $n \rightarrow \infty$, with the probability density function

$$
f_{Y}(y)=\frac{\sqrt{2}}{3^{1 / 4} \Gamma(1 / 4)} e^{-\frac{y^{4}}{12}} .
$$

Chatterjee and Shao [9] showed that the Berry-Esseen bound is of order $O\left(n^{-1 / 2}\right)$.

For the rest of this subsection, we consider only the case where $\beta=1$. Assume that conditions (B1)-(B3) are satisfied. Let $W=n^{\frac{1}{2 k}} m(\mathbf{X})$. Ellis and Newman [17] showed that $W$ converges weakly to a distribution with density

$$
p(y)=c_{1} \exp \left(-h^{(2 k)}(0) y^{2 k} /(2 k) !\right),
$$

where $c_{1}$ is a normalizing constant. For the concentration inequality, Chatterjee and Dey [8] used Stein's method to prove that for any $n \geq 1$ and $t \geq 0$,

$$
\mathrm{P}(|W| \geq t) \leq 2 e^{-c_{\rho} t^{2 k}},
$$

where $c_{\rho}>0$ is a constant depending only on $\rho$. Moreover, Shao and Zhang [26] proved the Berry-Esseen bound:

$$
\sup _{z \in \mathbb{R}}|\mathrm{P}(W \leq z)-\mathrm{P}(Y \leq z)| \leq C n^{-\frac{1}{2 k}},
$$

where $Y \sim p(y)$ as defined in (3.5) and $C>0$ is a constant.

In this subsection, we provide the Cramér-type moderate deviation for $W$.

Theorem 3.1. Let $W$ be defined as above. If $\beta=1$, we have

$$
\frac{\mathrm{P}(W>z)}{\mathrm{P}(Y>z)}=1+O(1) n^{-1 / k}\left(1+z^{2 k+2}\right),
$$

uniformly in $z \in\left(0, n^{\frac{1}{k(2 k+2)}}\right)$. 
Corollary 3.1. For the simple Curie-Weiss model, in which case $\rho=\frac{1}{2} \boldsymbol{\delta}_{1}+$ $\frac{1}{2} \boldsymbol{\delta}_{-1}$ and $\boldsymbol{\delta}$ is the Dirac measure. Then,

$$
\frac{\mathrm{P}(W>z)}{\mathrm{P}(Y>z)}=1+O(1) n^{-1 / 2}\left(1+z^{6}\right)
$$

uniformly in $z \in\left(0, n^{1 / 12}\right)$, where $Y \sim \mathcal{W}(4,12)$.

After we finished this paper, we learnt that Can and Pham [4] proved Corollary 3.1 by a completely different approach.

Remark 3.1. Comparing to Shao and Zhang [26, Theorem 3.2, (ii)], we assume the additional condition that $\rho$ is a symmetric measure. Following the proofs of Theorem 3.1 and Shao and Zhang [26, Theorem 3.2], we have (3.6) can be improved to

$$
\sup _{z \in \mathbb{R}}|\mathrm{P}(W \leq z)-\mathrm{P}(Y \leq z)| \leq C n^{-1 / k}
$$

\subsection{The imitative monomer-dimer mean-field model}

In this subsection, we consider the imitative monomer-dimer model and give the moderate deviation result. A pure monomer-dimer model can be used to study the properties of diatomic oxygen molecules deposited on tungsten or liquid mixtures with molecules of unequal size, see $[18,24]$ for example. Chang [5] studied the attractive component of the van der Waals potential, while Alberici, Contucci, Fedele and Mingione [1] and Alberici, Contucci and Mingione [2] considered the asymptotic properties.

Chen [15] recently obtained the Berry-Esseen bound by using Stein's method. In this subsection, we apply our main theorem to obtain the moderate deviation result.

For $n \geq 1$, let $G=(V, E)$ be a complete graph with vertex set $V=\{1, \ldots, n\}$ and edge set $E=\{u v=\{u, v\}: u, v \in V, u<v\}$. A dimer configuration on the graph $G$ is a set $D$ of pairwise nonincident edges satisfying the following rule: if $u v \in D$, then for all $w \neq v, u w \notin D$. Given a dimer configuration $D$, the set of monomers $\mathcal{M}(D)$ is the collection of dimer-free vertices. Let $\mathbf{D}$ denote the set of all dimer configurations. Denote the number of elements by \#(·). Then, we have

$$
2 \#(D)+\#(\mathcal{M}(D))=n
$$


Q.M. Shao, M.C. Zhang and Z.S. Zhang/Moderate deviation for nonnormal approximatidwe

We now introduce the imitative monomer-dimer model. The Hamiltonian of the model with an imitation coefficient $J \geq 0$ and an external field $h \in \mathbb{R}$ is given by

$$
-T(D)=n\left(J m(D)^{2}+b m(D)\right)
$$

for all $D \in \mathbf{D}$, where $m(D)=\#(\mathcal{M}(D)) / n$ is called the monomer density and the parameter $b$ is given by

$$
b=\frac{\log n}{2}+h-J
$$

The associated Gibbs measure is defined as

$$
p(D)=\frac{e^{-T(D)}}{\sum_{D \in \mathbf{D}} e^{-T(D)}} .
$$

Let

$$
H(x)=-J x^{2}-\frac{1}{2}(1-g(\tau(x))+\log (1-g(\tau(x)))),
$$

where

$$
g(x)=\frac{1}{2}\left(\sqrt{e^{4 x}+4 e^{2 x}}-e^{2 x}\right), \quad \tau(x)=(2 x-1) J+h .
$$

Alberici, Contucci and Mingione [2] showed that the imitative monomer-dimer model exhibits the following three phases. Let

$$
J_{c}=\frac{1}{4(3-2 \sqrt{2})}, \quad h_{c}=\frac{1}{2} \log (2 \sqrt{2}-2)-\frac{1}{4} .
$$

There exists a function $\gamma:\left(J_{c}, \infty\right) \rightarrow \mathbb{R}$ with $\gamma\left(J_{c}\right)=h_{c}$ such that if $(J, h) \notin \Gamma$, where $\Gamma:=\left\{(J, \gamma(J)): J>J_{c}\right\}$, then the function $H(x)$ has a unique maximizer $m_{0}$ that satisfies $m_{0}=g\left(\tau\left(m_{0}\right)\right)$. Moreover, if $(J, h) \notin \Gamma \cup\left\{\left(J_{c}, h_{c}\right)\right\}$, then $H^{\prime \prime}\left(m_{0}\right)<0$. If $(J, h)=\left(J_{c}, h_{c}\right)$, then $m_{0}=m_{c}:=2-\sqrt{2}$ and

$$
H^{\prime}\left(m_{c}\right)=H^{\prime \prime}\left(m_{c}\right)=H^{(3)}\left(m_{c}\right)=0,
$$

but

$$
H^{(4)}\left(m_{c}\right)<0 .
$$

If $(J, h) \in \Gamma$, then $H(s)$ has two distinct maximizers; therefore, in this case, $m(D)$ may not converge. Hence, we consider only the cases when $(J, h) \notin \Gamma$.

Alberici, Contucci and Mingione [2] showed that when $(J, h) \notin \Gamma \cup\left\{\left(J_{c}, h_{c}\right)\right\}$, $n^{1 / 2}\left(m(D)-m_{0}\right)$ converges to a normal distribution with zero mean and variance 
Q.M. Shao, M.C. Zhang and Z.S. Zhang/Moderate deviation for nonnormal approximatidn

$\lambda_{0}=-\left(H^{\prime \prime}\left(m_{0}\right)\right)^{-1}-(2 J)^{-1}$. However, when $(J, h)=\left(J_{c}, h_{c}\right), n^{1 / 4}\left(m(D)-m_{0}\right)$ converges to $Y$ in distribution, whose p.d.f. is given by

$$
p(y)=c_{1} e^{-\lambda_{c} y^{4} / 24}
$$

with $\lambda_{c}=-H^{(4)}\left(m_{c}\right)>0$ and $c_{1}$ is a normalizing constant. Chen [15] obtained the Berry-Esseen bound using Stein's method.

We use the following notations. Let $\Sigma=\{0,1\}^{n}$. For each $\sigma=\left(\sigma_{1}, \ldots, \sigma_{n}\right) \in$ $\Sigma$, define a Hamiltonian

$$
-T(\sigma)=n\left(J m(\sigma)^{2}+b m(\sigma)\right)
$$

where $m(\sigma)=n^{-1}\left(\sigma_{1}+\cdots+\sigma_{n}\right)$ is the magnetization of the configuration $\sigma$. Denote by $\boldsymbol{A}(\sigma)$ the set of all sites $i \in V$ such that $\sigma_{i}=1$. Also, let $D(\sigma)$ denote the total number of dimer configurations $D \in \mathbf{D}$ with $\mathcal{M}(D)=\boldsymbol{A}(\sigma)$. Therefore, the Gibbs measure can be written as

$$
p(\sigma)=\frac{D(\sigma) \exp (-T(\sigma))}{\sum_{\tau \in \Sigma} D(\tau) \exp (-T(\tau))} .
$$

The following result gives a Cramér-type moderate deviation for the magnetization.

Theorem 3.2. If $(J, h) \notin \Gamma \cup\left\{J_{c}, h_{c}\right\}$, then, for $0 \leq z \leq n^{1 / 6}$,

$$
\frac{\mathrm{P}\left(n^{1 / 2}\left(m(\sigma)-m_{0}\right)>z\right)}{\mathrm{P}\left(Z_{0}>z\right)}=1+O(1) n^{-1 / 2}\left(1+z^{3}\right),
$$

where $Z_{0}$ follows normal distribution with zero mean and variance $\lambda_{0}=-\left(H^{\prime \prime}\left(m_{0}\right)\right)^{-1}-$ $(2 J)^{-1}$. If $(J, h)=\left(J_{c}, h_{c}\right)$, then for $0 \leq z \leq n^{1 / 20}$,

$$
\frac{\mathrm{P}\left(n^{1 / 4}\left(m(\sigma)-m_{c}\right)>z\right)}{\mathrm{P}(Y>z)}=1+O(1) n^{-1 / 4}\left(1+z^{5}\right),
$$

where $Y$ is a random variable with the probability density function given in (3.8).

\section{Proofs of main results}

In this section, we give the proofs of the main theorems. In what follows, we use $C$ or $C_{1}, C_{2}, \ldots$ to denote a finite constant depending only on $c_{1}, c_{2}, c_{3}, d_{0}, d_{1}, \tau_{1}, \tau_{2}, \mu_{1}$ and $\alpha$, where $\mu_{1}=\max (g(1),|g(-1)|)+1$, and $C$ might be different in different places. 


\subsection{Proof of Theorem 2.1}

Let $Y$ be a random variable with a probability density function given in (2.4) and $F(z)$ be the distribution function of $Y$. We start with a preliminary lemma on the properties of $(1-F(w)) / p(w)$ and $F(w) / p(w)$, whose proof is postponed to Subsection 4.2.

Lemma 4.1. Assume that conditions (A1)-(A4) are satisfied. Then, we have

$$
\frac{1}{\max \left(1, c_{3}\right)(1+g(w))} \leq \frac{1-F(w)}{p(w)} \leq \min \left\{\frac{1}{g(w)}, 1 / c_{1}\right\} \text { for } w>0
$$

and

$$
\frac{F(w)}{p(w)} \leq \min \left\{\frac{1}{|g(w)|}, 1 / c_{1}\right\} \text { for } w<0 .
$$

Let $f_{z}$ be the solution to Stein's equation

$$
f^{\prime}(w)-f(w) g(w)=I(w \leq z)-F(z) .
$$

As shown in Chatterjee and Shao [9], the solution $f_{z}$ can be written as

$$
f_{z}(w)= \begin{cases}\frac{F(w)(1-F(z))}{p(w)}, & w \leq z \\ \frac{F(z)(1-F(w))}{p(w)}, & w>z\end{cases}
$$

Let

$$
\begin{aligned}
& I_{1}=\mathrm{E}\left(\int_{|t| \leq \delta}\left|f_{z}(W+t) g(W+t)-f_{z}(W) g(W)\right| \hat{K}(t) d t\right), \\
& I_{2}=\mathrm{E}\left(\left|\left(\mathrm{E}\left(\hat{K}_{1} \mid W\right)-1\right) f_{z}(W) g(W)\right|\right), \\
& I_{3}=\mathrm{E}\left(\left|\left(\mathrm{E}\left(\hat{K}_{1} \mid W\right)-1\right)(P(Y>z)-I(W>z+\delta))\right|\right), \\
& I_{4}=\mathrm{E}\left(f_{z}(W)|R(W)|\right) .
\end{aligned}
$$

The following propositions provide estimates of $I_{1}, I_{2}, I_{3}$ and $I_{4}$, whose proofs are given in Subsection 4.4 .

Proposition 4.1. If $\delta \leq 1$, then

$$
I_{1} \leq C \delta
$$

Assume that $z \geq 0, \max \left(\delta, \delta_{1}, \delta_{2}\right) \leq 1$ and $\delta z g^{2}(z)+\delta_{1} z g^{\tau_{1}+1}(z)+\delta_{2} z g^{\tau_{2}}(z) \leq$ 1. Then, we have

$$
I_{1} \leq C \delta\left(1+z g^{2}(z)\right)(1-F(z))
$$


Q.M. Shao, M.C. Zhang and Z.S. Zhang/Moderate deviation for nonnormal approximatidis

Proposition 4.2. We have

$$
I_{2}+I_{3} \leq C \delta_{1}, \quad I_{4} \leq C \delta_{2} .
$$

For $z>0, \max \left(\delta, \delta_{1}, \delta_{2}\right) \leq 1$ and $\delta z g^{2}(z)+\delta_{1} z g^{\tau_{1}+1}(z)+\delta_{2} z g^{\tau_{2}}(z) \leq 1$, we have

$$
\begin{aligned}
I_{2}+I_{3} & \leq C \delta_{1}\left(1+z g^{\tau_{1}+1}(z)\right)(1-F(z)), \\
I_{4} & \leq C \delta_{2}\left(1+z g^{\tau_{2}}(z)\right)(1-F(z)) .
\end{aligned}
$$

We are ready to give the proof of Theorem 2.1.

Proof of Theorem 2.1. From (2.1), we have

$$
\begin{aligned}
\mathrm{E}\left(f_{z}(W) g(W)-f_{z}(W) R(W)\right) \\
=\mathrm{E}\left(\int_{|t| \leq \delta} f_{z}^{\prime}(W+t) \hat{K}(t) d t\right) \\
=\mathrm{E}\left(\int _ { | t | \leq \delta } \left(f_{z}(W+t) g(W+t)\right.\right. \\
\quad+\mathrm{P}(Y>z)-I(W+t>z)) \hat{K}(t) d t) \\
\leq \mathrm{E}\left(\int_{|t| \leq \delta}\left(f_{z}(W+t) g(W+t)-f_{z}(W) g(W)\right) \hat{K}(t) d t\right) \\
+\mathrm{E}\left(\hat{K}_{1} f_{z}(W) g(W)\right) \\
+\mathrm{E}\left(\hat{K}_{1}(\mathrm{P}(Y>z)-I(W>z+\delta))\right) \\
\leq \mathrm{E}\left(\int_{|t| \leq \delta}\left|f_{z}(W+t) g(W+t)-f_{z}(W) g(W)\right| \hat{K}(t) d t\right) \\
+\mathrm{E}\left(\hat{K}_{1} f_{z}(W) g(W)\right) \\
+\mathrm{E}\left(\left|\mathrm{E}\left(\hat{K}_{1} \mid W\right)-1\right||P(Y>z)-I(W>z+\delta)|\right) \\
+\mathrm{P}(Y>z)-\mathrm{P}(W>z+\delta) .
\end{aligned}
$$

Rearranging (4.11) leads to

$$
\mathrm{P}(W>z+\delta)-\mathrm{P}(Y>z) \leq I_{1}+I_{2}+I_{3}+I_{4},
$$

where $I_{1}, I_{2}, I_{3}$ and $I_{4}$ are defined as in (4.5).

First, we use (4.12) and Propositions 4.1 and 4.2 to prove the Berry-Esseen bound

$$
|\mathrm{P}(W>z)-\mathrm{P}(Y>z)| \leq C\left(\delta+\delta_{1}+\delta_{2}\right)
$$


Q.M. Shao, M.C. Zhang and Z.S. Zhang/Moderate deviation for nonnormal approximatidra

where $C \geq 1$. By (4.6), (4.8) and (4.12), for $\delta \leq 1$, we have

$$
\mathrm{P}(W>z+\delta)-P(Y>z) \leq C\left(\delta+\delta_{1}+\delta_{2}\right)
$$

Together with

$$
\mathrm{P}(Y>z)-\mathrm{P}(Y>z+\delta) \leq c_{1} \int_{z}^{z+\delta} e^{-G(w)} d w \leq c_{1} \delta
$$

we have

$$
\mathrm{P}(W>z)-\mathrm{P}(Y>z) \leq C\left(\delta+\delta_{1}+\delta_{2}\right) .
$$

Similarly, we have

$$
\mathrm{P}(W>z)-\mathrm{P}(Y>z) \geq-C\left(\delta+\delta_{1}+\delta_{2}\right) .
$$

This proves the inequality (4.13) for $\delta \leq 1$. For $\delta>1$, (4.13) is trivial because $C \geq 1$.

Next, we move to prove (2.12). Let $z_{0}>1$ be a constant such that

$$
\min \left\{z_{0} g^{2}\left(z_{0}\right), z_{0} g^{\tau_{1}+1}\left(z_{0}\right), z_{0} g^{\tau_{2}}\left(z_{0}\right), z_{0}\right\} \geq 1 .
$$

For $0 \leq z \leq z_{0}$, (2.12) follows from (4.13) because

$$
\frac{\mathrm{P}(W>z)-\mathrm{P}(Y>z)}{\mathrm{P}(Y>z)} \leq \frac{C\left(\delta+\delta_{1}+\delta_{2}\right)}{1-F\left(z_{0}\right)}
$$

where $C$ is a constant.

For $z>z_{0}$, and thus $z>1$, we can assume $\max \left\{\delta, \delta_{1}, \delta_{2}\right\} \leq 1$; otherwise, it would contradict the condition

$$
\delta z g^{2}(z)+\delta_{1} z g^{\tau_{1}+1}(z)+\delta_{2} z g^{\tau_{2}}(z) \leq 1 .
$$

In this case, it follows that

$$
\delta \leq 1, \quad \delta g^{2}(z) \leq \delta z g^{2}(z) \leq 1
$$

provided that (4.16) holds.

By (4.12) and Propositions 4.1 and 4.2,

$$
\begin{aligned}
& \mathrm{P}(W>z+\delta)-(1-F(z)) \\
& \quad \leq I_{1}+I_{2}+I_{3}+I_{4} \\
& \leq C(1-F(z))\left(\delta\left(1+z g^{2}(z)\right)\right. \\
& \left.\quad+\delta_{1}\left(1+z g^{\tau_{1}+1}(z)\right)+\delta_{2}\left(1+z g^{\tau_{2}}(z)\right)\right) .
\end{aligned}
$$


Q.M. Shao, M.C. Zhang and Z.S. Zhang/Moderate deviation for nonnormal approximatidh5

By replacing $z$ with $z-\delta$, and noting that $g$ is nondecreasing, we can rewrite (4.18) as

$$
\begin{aligned}
\mathrm{P}(W>z)-(1-F(z-\delta)) & \\
\leq C(1-F(z-\delta))( & \delta\left(1+z g^{2}(z)\right)+\delta_{1}\left(1+z g^{\tau_{1}+1}(z)\right) \\
& \left.+\delta_{2}\left(1+z g^{\tau_{2}}(z)\right)\right)
\end{aligned}
$$

As $p(y)$ is decreasing in $[z-\delta, z]$, we have

$$
\begin{aligned}
F(z)-F(z-\delta) & =\int_{z-\delta}^{z} p(t) d t \\
& \leq \delta p(z-\delta) \leq e^{\delta g(z)} \delta p(z) .
\end{aligned}
$$

By (4.17), it follows that $\delta g(z) \leq(1 / 2) \delta\left(1+g^{2}(z)\right) \leq 1$. By $(4.1)$, we also have

$$
p(z) \leq \max \left(1, c_{3}\right)(1+g(z))(1-F(z))
$$

then,

$$
F(z)-F(z-\delta) \leq C \delta(1+g(z))(1-F(z))
$$

for some constant $C$. Recall that $\delta(1+g(z)) \leq 2$; then,

$$
1-F(z-\delta) \leq C(1-F(z))
$$

Together with (4.19), we get

$$
\begin{aligned}
& \mathrm{P}(W>z)-(1-F(z)) \\
& \leq \mathrm{P}(W>z)-(1-F(z-\delta))+F(z)-F(z-\delta) \\
& \leq C(1-F(z-\delta))\left(\delta\left(1+z g^{2}(z)\right)+\delta_{1}\left(1+z g^{\tau_{1}+1}(z)\right)+\delta_{2}\left(1+z g^{\tau_{2}}(z)\right)\right) \\
& \quad+C \delta(1+g(z))(1-F(z)) \\
& \leq C(1-F(z))\left(\delta\left(1+z g^{2}(z)\right)+\delta_{1}\left(1+z g^{\tau_{1}+1}(z)\right)+\delta_{2}\left(1+z g^{\tau_{2}}(z)\right)\right) .
\end{aligned}
$$

Similarly, we can prove the lower bound as follows:

$$
\begin{aligned}
& \mathrm{P}(W>z)-(1-F(z)) \\
& \quad \geq-C(1-F(z))\left(\delta\left(1+z g^{2}(z)\right)+\delta_{1}\left(1+z g^{\tau_{1}+1}(z)\right)+\delta_{2}\left(1+z g^{\tau_{2}}(z)\right)\right) .
\end{aligned}
$$

This completes the proof of Theorem 2.1.

imsart-generic ver. 2020/08/06 file: AAP1589-final.tex date: April 28, 2021 


\subsection{Proof of Lemma 4.1}

For $w \geq 0$, by the monotonicity of $g(\cdot)$, we have

$$
\begin{aligned}
1-F(w) & =\int_{w}^{\infty} p(t) d t \\
& =c_{1} \int_{w}^{\infty} e^{-G(t)} d t \\
& =c_{1} \int_{w}^{\infty} \frac{1}{g(t)} e^{-G(t)} d G(t) \\
& \leq \frac{c_{1}}{g(w)} e^{-G(w)} \\
& =\frac{p(w)}{g(w)} .
\end{aligned}
$$

Let $H(w)=1-F(w)-p(w) / c_{1}$; then,

$$
H^{\prime}(w)=p(w)\left(g(w) / c_{1}-1\right) .
$$

Note that $g(w) / c_{1}=1$ has at most one solution in $(0,+\infty)$ and that $g(0)=0$; then, $H(w)$ takes the maximum at either 0 or $+\infty$. We have

$$
H(w) \leq \max \left\{H(0), \lim _{w \rightarrow \infty} H(w)\right\} \leq 0 .
$$

This proves the upper bound of (4.1). The inequality (4.2) can be obtained similarly.

To finish the proof, we need to prove that for $w \geq 0$,

$$
\frac{p(w)}{1+g(w)} \leq \max \left(1, c_{3}\right)(1-F(w)) .
$$

Let

$$
\zeta(w)=\frac{1}{1+g(w)} e^{-G(w)} .
$$

As $g^{\prime}(w) \leq c_{3}(1+g(w))$, we have

$$
-\zeta^{\prime}(w)=\frac{g(w)}{1+g(w)} e^{-G(w)}+\frac{g^{\prime}(w)}{(1+g(w))^{2}} e^{-G(w)} \leq \max \left(1, c_{3}\right) e^{-G(w)} .
$$

As $g(w)$ is nondecreasing and $g(w)>0$ for $w>0$, then $G(w)=\int_{0}^{w} g(t) d t \rightarrow \infty$ as $w \rightarrow \infty$. Therefore, $\lim _{w \rightarrow \infty} p(w)=0$. Taking the integration on both sides yields

$$
\zeta(w)=-\int_{w}^{\infty} \zeta^{\prime}(t) d t \leq \max \left(1, c_{3}\right) \int_{w}^{\infty} e^{-G(t)} d t
$$

which leads to (4.20). This completes the proof. 
Q.M. Shao, M.C. Zhang and Z.S. Zhang/Moderate deviation for nonnormal approximatidn

\subsection{Preliminary lemmas}

To prove Propositions 4.1 and 4.2, we first present some preliminary lemmas. Throughout this subsection, we assume that conditions (A1)-(A4) are satisfied.

Lemma 4.2. Assume that $0<\delta \leq 1$. Then, we have

$$
\sup _{|t| \leq \delta}|g(w+t)| \leq c_{2}\left(|g(w)|+\mu_{1}\right)
$$

where $\mu_{1}=\max (g(1),|g(-1)|)+1$.

Also, for $w>s>0$ and any positive number $a>1$, there exists $b(a)$ depending on $a, c_{2}$ and $c_{3}$, such that

$$
g(w)-g(w-s) \leq \frac{1}{a} g(w)+b(a)(g(s)+1),
$$

where one can choose

$$
b(a)=\left(\left(2 c_{2}\right)+\cdots+\left(2 c_{2}\right)^{m(a)}\right)+1 / a,
$$

and $m(a)=\left[\log _{2}\left(a c_{3}+1\right)\right]+1$.

Proof of Lemma 4.2. The inequality (4.22) can be derived immediately from (2.5). Meanwhile, (4.23) remains to be shown. For $a>1$, consider two cases.

Case 1. If $s<w \leq\left(a c_{3}+1\right) s$, denote $m:=m(a)=\left[\log _{2}\left(a c_{3}+1\right)\right]+1$. As $g$ is nondecreasing and by (2.5), we have

$$
g(w) \leq g\left(2^{m} s\right) \leq 2 c_{2} g\left(2^{m-1} s\right)+c_{2} .
$$

By induction, we have

$$
\begin{aligned}
g(w) & \leq\left(2 c_{2}\right)^{m} g(s)+c_{2}\left(1+\left(2 c_{2}\right)+\cdots+\left(2 c_{2}\right)^{m-1}\right) \\
& \leq b(a)(g(s)+1),
\end{aligned}
$$

where $b(a)=2 c_{2}\left(1+\left(2 c_{2}\right)+\cdots+\left(2 c_{2}\right)^{m(a)-1}\right)+1 / a$.

Case 2. If $w>\left(a c_{3}+1\right) s$, by (2.6), we have

$$
\begin{aligned}
g(w)-g(w-s) & =\int_{0}^{s} g^{\prime}(w-t) d t \\
& \leq c_{3} \int_{0}^{s} \frac{1+g(w-t)}{1+(w-t)} d t \\
& \leq \frac{1}{a}(g(w)+1) .
\end{aligned}
$$

imsart-generic ver. 2020/08/06 file: AAP1589-final.tex date: April 28, 2021 
By (4.24) and (4.25), this completes the proof.

Lemma 4.3. For $w \geq 0$ and any $a>0$, we have

$$
g^{\prime}(w) \leq \frac{1}{a} g(w)+c_{3}\left(g\left(a c_{3}\right)+1\right)+1 / a
$$

Proof of Lemma 4.3. Recall that (2.6) states that for $w \geq 0$,

$$
g^{\prime}(w) \leq c_{3}\left(\frac{1+g(w)}{1+w}\right)
$$

Fix $a>0$. When $w>a c_{3}$, we have

$$
g^{\prime}(w) \leq \frac{1}{a}(g(w)+1)
$$

When $w \leq a c_{3}$, by the monotonicity property of $g$, we have

$$
g^{\prime}(w) \leq c_{3}\left(g\left(a c_{3}\right)+1\right) .
$$

This completes the proof.

For $s>0$, define

$$
f(w, s)= \begin{cases}e^{G(w)-G(w-s)}-1, & w>s \\ e^{G(w)}-1, & 0 \leq w \leq s \\ 0, & w \leq 0 .\end{cases}
$$

We next consider a ratio property of $f(w, s)$. It is easy to see that $f(w, s)$ is absolutely continuous with respect to both $w$ and $s$, and the partial derivatives are

$$
\begin{aligned}
\frac{\partial}{\partial w} f(w, s)= & e^{G(w)-G(w-s)}(g(w)-g(w-s)) I(w>s) \\
& +e^{G(w)} g(w) I(0 \leq w \leq s)
\end{aligned}
$$

and

$$
\frac{\partial}{\partial s} f(w, s)=e^{G(w)-G(w-s)} g(w-s) I(0<s \leq w) .
$$

Lemma 4.4. Let $f(w):=f(w, s)$ be defined as in (4.27). For $0 \leq \delta \leq 1$ and $\delta|g(w)| \leq d_{1}$, we have

$$
\sup _{|u| \leq \delta}\left|\frac{f(w+u)+1}{f(w)+1}\right| I(w+u \geq 0) \leq \mu_{2}
$$

where $\mu_{2}=\exp \left(c_{2}\left(d_{1}+\mu_{1}\right)+\mu_{1}\right)$. Moreover, we have

$$
\sup _{|u| \leq \delta}\left|f^{\prime \prime}(w+u)\right| \leq \mu_{3}\left(g^{2}(w)+1\right)(f(w)+1) .
$$

where $\mu_{3}=2 c_{2}^{2}\left(c_{3}+1\right)\left(\mu_{1}^{2}+1\right) \mu_{2}$. 
Proof. Recall that $\mu_{1}=\max (g(1),|g(-1)|)+1$. When $w+u \geq 0$ and $w \geq 0$, as $g$ is nondecreasing, we have

$$
\begin{aligned}
\sup _{|u| \leq \delta}\left|\frac{f(w+u)+1}{f(w)+1}\right| & \leq e^{G(w+\delta)-G(w)} \\
& \leq e^{\delta|g(w+\delta)|} \leq e^{c_{2}\left(d_{1}+\mu_{1}\right)}
\end{aligned}
$$

where in the last inequality we use (4.22). When $w+u \geq 0, w<0$ and $|u| \leq \delta$, we have $0 \leq w+u<\delta \leq 1$; hence, by the nondecreasing property of $g$,

$$
\sup _{|u| \leq \delta}\left|\frac{f(w+u)+1}{f(w)+1}\right| \leq \sup _{|u| \leq \delta} e^{G(w+u)} \leq e^{G(\delta)} \leq e^{\mu_{1}} .
$$

This proves (4.30).

For $f^{\prime \prime}(w)$, by (4.28),

$$
\begin{aligned}
f^{\prime \prime}(w)= & e^{G(w)-G(w-s)}(g(w)-g(w-s))^{2} I(w>s) \\
& +e^{G(w)-G(w-s)}\left(g^{\prime}(w)-g^{\prime}(w-s)\right) I(w>s) \\
& +e^{G(w)} g^{2}(w) I(0 \leq w \leq s) \\
& +e^{G(w)} g^{\prime}(w) I(0 \leq w \leq s) .
\end{aligned}
$$

As $g$ is nondecreasing, we have $g^{\prime}(w-s) \geq 0$; thus, $g^{\prime}(w)-g^{\prime}(w-s) \leq g^{\prime}(w)$. For $w>s, 0 \leq g(w)-g(w-s) \leq g(w)$. Therefore,

$$
f^{\prime \prime}(w) \leq\left(g^{\prime}(w)+g^{2}(w)\right)(f(w)+1) I(w \geq 0) .
$$

By (2.6), for $c_{3}>1$, we have

$$
g^{2}(w)+g^{\prime}(w) \leq g^{2}(w)+c_{3}(1+g(w)) \leq 2\left(c_{3}+1\right)\left(g^{2}(w)+1\right) .
$$

Hence,

$$
f^{\prime \prime}(w) \leq 2\left(c_{3}+1\right)\left(g^{2}(w)+1\right)(f(w)+1) .
$$

By (4.22) and (4.30), we have

$$
\sup _{|u| \leq \delta}\left|f^{\prime \prime}(w+u)\right| \leq \mu_{3}\left(g^{2}(w)+1\right)(f(w)+1),
$$

where $\mu_{3}=2 c_{2}^{2}\left(c_{3}+1\right)\left(\mu_{1}^{2}+1\right) \mu_{2}$. This completes the proof of Lemma 4.4.

Let $W$ be the random variable defined as in Theorem 2.1. For $0 \leq \tau \leq$ $\max \left(2, \tau_{1}+1, \tau_{2}\right)$ and $s>0$, Lemmas 4.5 and 4.6 give the properties of $\mathrm{E}|g(W)|^{\tau}$, $\mathrm{E}|g(W)|^{\tau} e^{G(W)} I(0 \leq W \leq s)$ and $\mathrm{E}|g(W)|^{\tau} e^{G(W)-G(W-s)} I(W>s)$, which play a key role in the proofs of Propositions 4.1 and 4.2 .

imsart-generic ver. 2020/08/06 file: AAP1589-final.tex date: April 28, 2021 
Q.M. Shao, M.C. Zhang and Z.S. Zhang/Moderate deviation for nonnormal approximati@0

Lemma 4.5. Suppose that conditions (A1)-(A4) and (2.9)-(2.11) are satisfied with $\delta \leq 1$. For $0 \leq \tau \leq \max \left(2, \tau_{1}+1, \tau_{2}\right)$, we have

$$
\mathrm{E}|g(W)|^{\tau} \leq C
$$

Moreover, for $s>0$, we have

$$
\mathrm{E}\left(e^{G(W)-G(W-s)} g^{\tau}(W) I(W>s)\right) \leq C\left(1+g^{\tau}(s)\right)(\mathrm{E}(f(W, s))+1),
$$

and

$$
\mathrm{E}\left(e^{G(W)} g^{\tau}(W) I(0 \leq W \leq s)\right) \leq C\left(1+g^{\tau}(s)\right)(\mathrm{E}(f(W, s))+1) .
$$

Proof of Lemma 4.5. In this proof, we always assume that $\delta \leq 1$.

We first prove (4.32). Without loss of generality, we consider only the case where $\tau \geq 2$. As $\delta|g(W)| \leq d_{1}$, we have $\mathrm{E}|g(W)|^{\tau}<\infty$. To bound $\mathrm{E}|g(W)|^{\tau}$, without loss of generality, we consider only $\mathrm{E}^{\tau}(W) I(W \geq 0)$. Let $g_{+}(w):=$ $g(w) I(w \geq 0)$. As $g(0)=0$ and $g$ is differentiable, we find that $g_{+}(w)$ is absolutely continuous. By (2.1), we have

$$
\begin{aligned}
\mathrm{E}\left\{g^{\tau}(W) I(W \geq 0)\right\} & =\mathrm{E}\left\{g(W) \cdot g_{+}^{\tau-1}(W)\right\} \\
& :=Q_{1}+Q_{2},
\end{aligned}
$$

where

$$
\begin{aligned}
& Q_{1}=(\tau-1) \mathrm{E} \int_{|u| \leq \delta} g_{+}^{\tau-2}(W+u) g^{\prime}(W+u) I(W+u \geq 0) \hat{K}(u) d u \\
& Q_{2}=\mathrm{E} R(W) g_{+}^{\tau-1}(W) .
\end{aligned}
$$

The following inequality is well known: for any $a>0, x, y \geq 0$ and $\tau>1$

$$
x^{\tau-1} y \leq \frac{\tau-1}{a \tau} x^{\tau}+\frac{a^{\tau-1}}{\tau} y^{\tau} .
$$

For the first term $Q_{1}$, by (2.6), we have

$$
g^{\prime}(w+u) \leq c_{3}(1+|g(w+u)|) .
$$


Thus, for $w+u \geq 0$,

$$
\begin{aligned}
\sup _{|u| \leq \delta} & g_{+}^{\tau-2}(w+u) g^{\prime}(w+u) \\
& \leq c_{3} \sup _{|u| \leq \delta}\left(g_{+}^{\tau-1}(w+u)+g_{+}^{\tau-2}(w+u)\right) \\
& \leq 2 c_{3} \sup _{|u| \leq \delta}\left(g_{+}^{\tau-1}(w+u)+1\right) \\
& \leq \frac{1-\alpha}{8 \times\left(2 c_{2}\right)^{\tau} d_{0}(\tau-1)} \sup _{|u| \leq \delta}|g(w+u)|^{\tau}+D_{1,0},
\end{aligned}
$$

where we use (4.36) with

$$
a=\frac{8 \times\left(2 c_{2}\right)^{\tau+1} d_{0}(\tau-1)}{1-\alpha} \text { and } x=\left|g_{+}(w+u)\right|
$$

in the last inequality. Here and in the sequel, $D_{1,0}, D_{2,0}$, etc. denote constants depending on $c_{2}, c_{3}, d_{0}, d_{1}, \mu_{1}, \alpha$ and $\tau$. By (4.22), we have

$$
\sup _{|u| \leq \delta}|g(w+u)|^{\tau} \leq\left(2 c_{2}\right)^{\tau}\left(|g(w)|^{\tau}+\mu_{1}^{\tau}\right) .
$$

Then, by (2.9), we have

$$
Q_{1} \leq \frac{1-\alpha}{8} \mathrm{E}|g(W)|^{\tau}+D_{2,0}
$$

For $Q_{2}$, by (2.11) and using (4.36) again, we have

$$
Q_{2} \leq \alpha \mathrm{E} g_{+}^{\tau}(W)+\frac{1-\alpha}{4} \mathrm{E} g_{+}^{\tau}(W)+\left(\frac{4}{1-\alpha}\right)^{\tau-1}
$$

Hence, by (4.35), (4.37) and (4.38), we have

$$
\mathrm{E} g_{+}^{\tau}(W) \leq \frac{1}{6} \mathrm{E}|g(W)|^{\tau}+D_{3,0} .
$$

Similarly, we have

$$
\mathrm{E} g_{-}^{\tau}(W) \leq \frac{1}{6} \mathrm{E}|g(W)|^{\tau}+D_{4,0}
$$

Combining the two foregoing inequalities yields (4.32).

As to (4.33) and (4.34), we first consider the case where $\tau \geq 2$. Write $f(w):=$ $f(w, s)$. By $(2.1)$ and (4.28), we have

$$
\begin{aligned}
\mathrm{E}\left(g(W)^{\tau} f(W)\right) & =\mathrm{E} g(W)\left\{g(W)^{\tau-1} f(W)\right\} \\
& =M_{1}+M_{2}+M_{3}+M_{4},
\end{aligned}
$$


Q.M. Shao, M.C. Zhang and Z.S. Zhang/Moderate deviation for nonnormal approximatid2

where

$$
\begin{aligned}
& M_{1}=\mathrm{E} \int_{|u| \leq \delta} g^{\tau}(W+u) e^{G(W+u)} I(0 \leq W+u \leq s) \hat{K}(u) d u, \\
& M_{2}=\mathrm{E} \int_{|u| \leq \delta} g^{\tau-1}(W+u)(g(W+u)-g(W+u-s)) \\
& \quad \times e^{G(W+u)-G(W+u-s)} I(W+u>s) \hat{K}(u) d u, \\
& M_{3}=(\tau-1) \mathrm{E} \int_{|u| \leq \delta} g^{\tau-2}(W+u) g^{\prime}(W+u) f(W+u) \hat{K}(u) d u, \\
& M_{4}=\mathrm{E} R(W) g^{\tau-1}(W) f(W) .
\end{aligned}
$$

We next give the bounds of $M_{1}, M_{2}, M_{3}$ and $M_{4}$. For $M_{1}$, by (2.9) and (4.30) and noting that $g$ is nondecreasing, we have

$$
\begin{aligned}
M_{1} & \leq d_{0} g^{\tau}(s) \mathrm{E} \sup _{|u| \leq \delta}(f(W+u)+1) I(0 \leq W+u \leq s) \\
& \leq d_{0} \mu_{2} g^{\tau}(s) \mathrm{E}(f(W)+1) .
\end{aligned}
$$

To bound $M_{2}$, we first give the bound of $g(w+u)$ and $g(w+u)-g(w+u-s)$ for $|u| \leq \delta$. By (4.22), we have

$$
\sup _{|u| \leq \delta}|g(w+u)| \leq c_{2}\left(|g(w)|+\mu_{1}\right)
$$

Furthermore, by (4.23) with $a=2^{\tau+2} d_{0} \mu_{2} c_{2}^{\tau} /(1-\alpha)$, for $w+u>s$, there exists a constant $D_{1}$ depending on $c_{2}, c_{3}, d_{0}, d_{1}, \mu_{1}, \alpha$ and $\tau$ such that

$$
\begin{aligned}
& \sup _{|u| \leq \delta}|g(w+u)-g(w+u-s)| \\
& \quad \leq \frac{1-\alpha}{2^{\tau+3} d_{0} \mu_{2} c_{2}^{\tau}} \sup _{|u| \leq \delta}|g(w+u)|+D_{1}(g(s)+1) .
\end{aligned}
$$

By (4.36), (4.42) and (4.43), we have

$$
\begin{aligned}
\sup _{|u| \leq \delta} & \left|g(W+u)^{\tau-1}(g(W+u)-g(W+u-s))\right| \\
& \leq\left(\frac{1-\alpha}{2^{\tau+3} d_{0} \mu_{2} c_{2}^{\tau}} \sup _{|u| \leq \delta}|g(W+u)|+D_{1}(g(s)+1)\right) \sup _{|u| \leq \delta}|g(W+u)|^{\tau-1} \\
& \leq \frac{1-\alpha}{2^{\tau+2} d_{0} \mu_{2} c_{2}^{\tau}} \sup _{|u| \leq \delta}|g(W+u)|^{\tau}+\frac{2^{\tau+3} d_{0} \mu_{2} c_{2}^{\tau}}{\tau(1-\alpha)} \times D_{1}^{\tau}(1+g(s))^{\tau} \\
& \leq \frac{1-\alpha}{4 d_{0} \mu_{2}}\left(|g(W)|^{\tau}+\mu_{1}^{\tau}\right)+\frac{2^{\tau+3} d_{0} \mu_{2} c_{2}^{\tau}}{\tau(1-\alpha)} \times D_{1}^{\tau}(1+g(s))^{\tau} \\
& \leq \frac{1-\alpha}{4 d_{0} \mu_{2}}|g(W)|^{\tau}+D_{2}\left(1+g^{\tau}(s)\right)
\end{aligned}
$$

imsart-generic ver. 2020/08/06 file: AAP1589-final.tex date: April 28, 2021 
Q.M. Shao, M.C. Zhang and Z.S. Zhang/Moderate deviation for nonnormal approximati@ß

where

$$
D_{2}=\frac{2^{2 \tau+3} d_{0} \mu_{2} c_{2}^{\tau}}{\tau(1-\alpha)} \times D_{1}^{\tau}+\frac{(1-\alpha) \mu_{1}^{\tau}}{4 d_{0} \mu_{2}}
$$

By (2.9) and (4.30), we have

$$
\begin{aligned}
M_{2} \leq & \frac{1-\alpha}{4} \mathrm{E}|g(W)|^{\tau}(f(W)+1) \\
& \quad+d_{0} \mu_{2} D_{2}\left(1+g^{\tau}(s)\right) \mathrm{E}(f(W)+1) .
\end{aligned}
$$

For $M_{3}$, by Lemma 4.3 and similar to (4.44), we have

$$
\begin{aligned}
M_{3} \leq & \frac{1-\alpha}{4} \mathrm{E}|g(W)|^{\tau}(f(W)+1) \\
& \quad+D_{3}\left(1+g^{\tau}(s)\right) \mathrm{E}(f(W)+1)
\end{aligned}
$$

where $D_{3}$ is a finite constant depending on $c_{2}, c_{3}, d_{0}, d_{1}, \mu_{1}, \alpha$ and $\tau$.

For $M_{4}$, by (2.11) and (4.36), we have

$$
\begin{aligned}
M_{4} & \leq \alpha \mathrm{E}|g(W)|^{\tau} f(W)+\alpha \mathrm{E}|g(W)|^{\tau-1} f(W) \\
& \leq\left(\alpha+\frac{1-\alpha}{4}\right) \mathrm{E}|g(W)|^{\tau} f(W)+\left(\frac{4 \alpha}{1-\alpha}\right)^{\tau-1} \mathrm{E} f(W) .
\end{aligned}
$$

By (4.39), (4.41) and (4.44)-(4.46), we have

$$
\begin{aligned}
\mathrm{E}|g(W)|^{\tau} f(W) \leq( & \left.\alpha+\frac{3(1-\alpha)}{4}\right) \mathrm{E}|g(W)|^{\tau} f(W) \\
& +\left(D_{4}+\mathrm{E}|g(W)|^{\tau}\right)\left(1+g^{\tau}(s)\right) \mathrm{E}(f(W)+1)
\end{aligned}
$$

where $D_{4}$ is a constant depending on $c_{2}, c_{3}, d_{0}, d_{1}, \mu_{1}, \alpha$ and $\tau$. Rearranging the inequality gives

$$
\mathrm{E}|g(W)|^{\tau} f(W) \leq \frac{4\left(D_{4}+\mathrm{E}|g(W)|^{\tau}\right)}{1-\alpha}\left(1+g^{\tau}(s)\right) \mathrm{E}(f(W)+1)
$$

Combining (4.32) and (4.47), we have

$$
\mathrm{E}|g(W)|^{\tau}(f(W)+1) \leq D_{5}\left(1+g^{\tau}(s)\right) \mathrm{E}(f(W)+1)
$$

where $D_{5}$ is a constant depending on $c_{2}, c_{3}, d_{0}, d_{1}, \mu_{1}, \alpha$ and $\tau$. This proves (4.33) and (4.34) for $\tau \geq 2$.

For $0 \leq \tau<2$ with $\mathrm{E}|g(W)|^{2}<\infty$. By the Cauchy inequality, we have

$$
\left(1+g^{2-\tau}(s)\right)|g(w)|^{\tau} \leq 1+g^{2}(s)+2 g^{2}(w)
$$


Q.M. Shao, M.C. Zhang and Z.S. Zhang/Moderate deviation for nonnormal approximatid4

and noting that for $s>0$ and $g(s)>0$,

$$
\begin{aligned}
|g(w)|^{\tau} & \leq \frac{1+g^{2}(s)+2 g^{2}(w)}{1+g^{2-\tau}(s)} \\
& \leq g^{\tau}(s)+\frac{1+2 g^{2}(w)}{1+g^{2-\tau}(s)} .
\end{aligned}
$$

By (4.48) with $\tau=2$, we have

$$
\mathrm{E}|g(W)|^{2}(f(W)+1) \leq D_{6}\left(1+g^{2}(s)\right) \mathrm{E}(f(W)+1),
$$

where $D_{6}$ is a constant depending on $c_{2}, c_{3}, d_{0}, d_{1}, \mu_{1}, \alpha$ and $\tau$.

Thus, for $0 \leq \tau<2$, by (4.49) and (4.50), we have

$$
\begin{aligned}
\mathrm{E}|g(W)|^{\tau}(f(W)+1) \leq & g^{\tau}(s) \mathrm{E}(f(W)+1) \\
& +\frac{\mathrm{E}(f(W)+1)+2 \mathrm{E} g^{2}(W)(f(W)+1)}{1+g^{2-\tau}(s)} \\
\leq & D_{7}\left(1+g^{\tau}(s)\right) \mathrm{E}(f(W)+1),
\end{aligned}
$$

where $D_{7}$ is a constant depending on $c_{2}, c_{3}, d_{0}, d_{1}, \mu_{1}, \alpha$ and $\tau$. This completes the proof together with (4.48).

Lemma 4.6. Let $f(w, s)$ be defined as in (4.27). Let $0<\delta \leq 1$ and $s>0$. Suppose that the conditions in Theorem 2.1 are satisfied. Then, we have

$$
\begin{aligned}
& \mathrm{E}(f(W, s)+1) \\
& \leq C(1+s) \exp \left\{C \left(\delta\left(1+s g^{2}(s)\right)+\delta_{1}\left(1+s g^{\tau_{1}+1}(s)\right)\right.\right. \\
& \left.\left.+\delta_{2}\left(1+s g^{\tau_{2}}(s)\right)\right)\right\} .
\end{aligned}
$$

Remark 4.1. Following the proof of Lemma 4.6, if we assume that the condition (2.7) is replaced by (2.18) and (2.19), then the result of Lemma 4.6 still holds.

Proof of Lemma 4.6. Let $h(s)=\mathrm{E} f(W, s)$ and let $f(w):=f(w, s)$. By (4.28) and (4.29), for $s>0$, we have

$$
\begin{aligned}
h^{\prime}(s) & =\mathrm{E}\left(e^{G(W)-G(W-s)} g(W-s) I(W>s)\right) \\
& =\mathrm{E}(f(W) g(W))+E(g(W) I(W>0))-E\left(f^{\prime}(W)\right) .
\end{aligned}
$$

We first show that $h^{\prime}(s)$ can be bounded by a function of $h(s)$. We then solve the differential inequality to obtain the bound of $h(s)$, using an idea similar to that in the proof of Lemma 4.5.

imsart-generic ver. 2020/08/06 file: AAP1589-final.tex date: April 28, 2021 
Q.M. Shao, M.C. Zhang and Z.S. Zhang/Moderate deviation for nonnormal approximati@5

By (2.1), we have

$$
\mathrm{E}(f(W) g(W))-\mathrm{E}\left(f^{\prime}(W)\right)=T_{1}+T_{2}+T_{3},
$$

where

$$
\begin{aligned}
& T_{1}=\mathrm{E}\left(\int_{|u| \leq \delta}\left(f^{\prime}(W+u)-f^{\prime}(W)\right) \hat{K}(u) d u\right), \\
& T_{2}=\mathrm{E} f^{\prime}(W)\left(\mathrm{E}\left(\hat{K}_{1} \mid W\right)-1\right), \\
& T_{3}=\mathrm{E}(f(W) R(W)) .
\end{aligned}
$$

We next give the bounds of $T_{1}, T_{2}$ and $T_{3}$.

i). The bound of $T_{1}$. By (4.31), we have

$$
\begin{aligned}
& \sup _{|u| \leq \delta}\left|f^{\prime}(w+u)-f^{\prime}(w)\right| \\
& \leq \delta \sup _{|u| \leq \delta}\left|f^{\prime \prime}(w+u)\right| \\
& \leq \delta \mu_{3}\left(g^{2}(w)+1\right)(f(w)+1) .
\end{aligned}
$$

By (2.9) and Lemma 4.5, we have

$$
\begin{aligned}
\left|T_{1}\right| & \leq \delta d_{0} \mu_{3} \mathrm{E}\left(g^{2}(W)+1\right)(f(W)+1) \\
& \leq D_{8} \delta\left(1+g^{2}(s)\right) \mathrm{E}(f(W)+1),
\end{aligned}
$$

where $D_{8}$ is a constant depending on $c_{2}, c_{3}, d_{0}, d_{1}, \mu_{1}$ and $\alpha$.

ii). The bound of $T_{2}$. By (2.7) and Lemma 4.5, we have

$$
\begin{aligned}
\left|T_{2}\right| & \leq \delta_{1} \mathrm{E}\left(|g(W)|\left(|g(W)|^{\tau_{1}}+1\right)(f(W)+1)\right) \\
& \leq 2 \delta_{1} \mathrm{E}\left(|g(W)|^{\tau_{1}+1}+1\right)(f(W)+1) \\
& \leq D_{9} \delta_{1}\left(1+g^{\tau_{1}+1}(s)\right) \mathrm{E}(f(W)+1),
\end{aligned}
$$

where $D_{9}$ is a constant depending on $c_{2}, c_{3}, d_{0}, d_{1}, \mu_{1}, \tau_{1}$ and $\alpha$.

iii). The bound of $T_{3}$. By (2.8) and Lemma 4.5, we have

$$
\begin{aligned}
T_{3} & \leq \delta_{2} \mathrm{E}\left(|g(W)|^{\tau_{2}}+1\right) f(W) \\
& \leq D_{10} \delta_{2}\left(1+g^{\tau_{2}}(s)\right) \mathrm{E}(f(W)+1),
\end{aligned}
$$

where $D_{10}$ is a constant depending on $c_{2}, c_{3}, d_{0}, d_{1}, \mu_{1}, \tau_{2}$ and $\alpha$.

imsart-generic ver. 2020/08/06 file: AAP1589-final.tex date: April 28, 2021 
Q.M. Shao, M.C. Zhang and Z.S. Zhang/Moderate deviation for nonnormal approximati26

By (4.32), we have

$$
\mathrm{E} g(W) I(W>0) \leq D_{11}
$$

where $D_{11}$ is a constant depending on $c_{2}, c_{3}, d_{0}, d_{1}, \mu_{1}$ and $\alpha$. By (4.52)-(4.56), we have

$$
\begin{aligned}
h^{\prime}(s) \leq D_{11}+D_{12} & \left(\delta\left(1+g^{2}(s)\right)+\delta_{1}\left(1+g^{\tau_{1}+1}(s)\right)+\delta_{2}\left(1+g^{\tau_{2}}(s)\right)\right) \\
\times & \mathrm{E}(f(W)+1),
\end{aligned}
$$

where $D_{12}=\max \left(D_{8}, D_{9}, D_{10}\right)$. Therefore,

$$
\begin{aligned}
h^{\prime}(s) \leq & D_{12}\left(\delta\left(1+g^{2}(s)\right)+\delta_{1}\left(1+g^{\tau_{1}+1}(s)\right)+\delta_{2}\left(1+g^{\tau_{2}}(s)\right)\right) h(s) \\
& +D_{11}+D_{12}\left(\delta\left(1+g^{2}(s)\right)+\delta_{1}\left(1+g^{\tau_{1}+1}(s)\right)+\delta_{2}\left(1+g^{\tau_{2}}(s)\right)\right),
\end{aligned}
$$

By solving the differential inequality and given that $s+s g^{\tau}(s) \leq 1+(1+$ $\left.g^{-\tau}(1)\right) \operatorname{sg}^{\tau}(s)$ for $\tau>0$ and $s \geq 0$, we have

$$
\begin{aligned}
\mathrm{E}(f(W)+1) \leq C_{1}(1+s) \exp \left\{C _ { 2 } \left(\delta\left(1+s g^{2}(s)\right)+\delta_{1}\left(1+s g^{\tau_{1}+1}(s)\right)\right.\right. \\
\left.\left.+\delta_{2}\left(1+s g^{\tau_{2}}(s)\right)\right)\right\}
\end{aligned}
$$

where $C_{1}$ and $C_{2}$ are constants depending on $c_{2}, c_{3}, d_{0}, d_{1}, \mu_{1}, \tau_{1}, \tau_{2}$ and $\alpha$. This completes the proof.

The next lemma gives the properties of the Stein solution.

Lemma 4.7. Let $f_{z}$ be the solution to Stein's equation (4.3). Then, for $z \geq 0$,

$$
\begin{gathered}
\left|f_{z}(w) g(w)\right| \leq \begin{cases}1-F(z), & w \leq 0 \\
F(z), & w>0\end{cases} \\
0 \leq f_{z}(w) \leq \begin{cases}(1-F(z)) / c_{1}, & w \leq 0 \\
F(z) / c_{1}, & w>0\end{cases}
\end{gathered}
$$

and

$$
\left|f_{z}^{\prime}(w)\right| \leq \begin{cases}2(1-F(z)), & w \leq 0 \\ 1, & 0<w \leq z \\ 2 F(z), & w>z\end{cases}
$$


Q.M. Shao, M.C. Zhang and Z.S. Zhang/Moderate deviation for nonnormal approximation

Proof of Lemma 4.7. Our first step is to prove (4.57). By (4.4), we have

$$
f_{z}(w) g(w)= \begin{cases}\frac{F(w) g(w)(1-F(z))}{p(w)}, & w \leq z, \\ \frac{F(z) g(w)(1-F(w))}{p(w)}, & w>z .\end{cases}
$$

Without loss of generality, we must consider only three case when $z>0$ :

1. $w<0$ : By $(4.2)$,

$$
\left|f_{z}(w) g(w)\right| \leq 1-F(z) .
$$

2. $0 \leq w \leq z$ : Since $w \leq z, 1-F(z) \leq 1-F(w)$, thus by (4.1),

$$
\left|f_{z}(w) g(w)\right| \leq \frac{F(w)|g(w)|(1-F(w))}{p(w)} \leq F(w) \leq F(z) .
$$

3. $w>z$ : By (4.1),

$$
\left|f_{z}(w) g(w)\right| \leq F(z)
$$

We can have a similar argument when $z \leq 0$, which completes the proof of (4.57). Additionally, (4.58) can be shown similarly. (4.59) follows directly from (4.3) and (4.57).

Lemma 4.8. For $z>0$ and $0 \leq \tau \leq \max \left(2, \tau_{1}+1, \tau_{2}\right)$,

$$
\mathrm{E}\left(f_{z}(W)|g(W)|^{\tau}\right) \leq C\left(1+z g^{\tau}(z)\right)(1-F(z)),
$$

provided that $\max \left(\delta, \delta_{1}, \delta_{2}\right) \leq 1$ and $\delta z g^{2}(z)+\delta_{1} z g^{\tau_{1}+1}(z)+\delta_{2} z g^{\tau_{2}}(z) \leq 1$.

Proof of Lemma 4.8. By (4.4),

$$
\mathrm{E}\left(f_{z}(W)|g(W)|^{\tau}\right)=T_{4}+T_{5}+T_{6},
$$

where

$$
\begin{aligned}
& T_{4}=F(z) \mathrm{E}\left(\frac{1-F(W)}{p(W)}|g(W)|^{\tau} I(W>z)\right), \\
& T_{5}=(1-F(z)) \mathrm{E}\left(\frac{F(W)}{p(W)}|g(W)|^{\tau} I(W<0)\right), \\
& T_{6}=(1-F(z)) \mathrm{E}\left(\frac{F(W)}{p(W)}|g(W)|^{\tau} I(0 \leq W \leq z)\right) .
\end{aligned}
$$

imsart-generic ver. 2020/08/06 file: AAP1589-final.tex date: April 28, 2021 
Q.M. Shao, M.C. Zhang and Z.S. Zhang/Moderate deviation for nonnormal approximati@8

i). For $T_{4}$, we first consider the case when $\tau \geq 1$. As $g(w)$ is increasing, $e^{G(w)-G(w-z)}$ is also increasing with respect to $w$; thus,

$$
I(W>z) \leq \frac{e^{G(W)-G(W-z)} I(W>z)}{e^{G(z)}} .
$$

By Lemma 4.6, we have $\max \left(\delta, \delta_{1}, \delta_{2}\right) \leq 1$ and $z$, satisfying that $\delta z g^{2}(z)+$ $\delta_{1} z g^{\tau_{1}+1}(z)+\delta_{2} z g^{\tau_{2}}(z) \leq 1$,

$$
\mathrm{E}(f(W, z)+1) \leq C(1+z) .
$$

Hence, by (4.1) and Lemma 4.5, we have

$$
\begin{aligned}
T_{4} & \leq C e^{-G(z)} \mathrm{E}|g(W)|^{\tau-1} e^{G(W)-G(W-z)} I(W>z) \\
& \leq C e^{-G(z)}\left(1+g^{\tau-1}(z)\right) \mathrm{E}(f(W, z)+1) \\
& \leq C e^{-G(z)}\left(1+z g^{\tau-1}(z)\right) \\
& \leq C\left(1+z g^{\tau}(z)\right)(1-F(z)),
\end{aligned}
$$

for $\max \left(\delta, \delta_{1}, \delta_{2}\right) \leq 1$ and $z$, satisfying that $\delta z g^{2}(z)+\delta_{1} z g^{\tau_{1}+1}(z)+\delta_{2} z g^{\tau_{2}}(z) \leq$ 1. If $0 \leq \tau<1$, then $g^{\tau}(w) \leq 2(1+g(w)) /\left(1+g^{1-\tau}(z)\right)$ for $w>z$. Therefore, (4.62) also holds for $0 \leq \tau<1$.

ii). As to $T_{5}$, because $F(w) / p(w) \leq 1 / c_{1}$ for $w \leq 0$,

$$
T_{5} \leq \frac{1}{c_{1}}(1-F(z)) \mathrm{E}|g(W)|^{\tau} I(W<0) .
$$

By (4.32), we have

$$
T_{5} \leq C(1-F(z))
$$

for some constant $C$.

iii). We now bound $T_{6}$. By Lemmas 4.5 and 4.6 ,

$$
\begin{aligned}
T_{6} & \leq C(1-F(z)) \mathrm{E} e^{G(W)}|g(W)|^{\tau} I(0 \leq W \leq z) \\
& \leq C(1-F(z))\left(1+g^{\tau}(z)\right) \mathrm{E} e^{G(W)} I(0 \leq W \leq z) \\
& \leq C(1-F(z))\left(1+z g^{\tau}(z)\right) .
\end{aligned}
$$

By (4.62)-(4.64), we have

$$
\mathrm{E}\left(f_{z}(W)|g(W)|^{\tau}\right) \leq C\left(1+z g^{\tau}(z)\right)(1-F(z)),
$$

which completes the proof. 
Q.M. Shao, M.C. Zhang and Z.S. Zhang/Moderate deviation for nonnormal approximati®9

\subsection{Proofs of Propositions 4.1 and 4.2}

We are now ready to give the proofs of Propositions 4.1 and 4.2 .

Proof of Proposition 4.1. Recalling (2.9), we have

$$
\begin{aligned}
I_{1} & \leq d_{0} \mathrm{E}\left(\sup _{|t| \leq \delta}\left|f_{z}(W+t) g(W+t)-f_{z}(W) g(W)\right|\right) \\
& \leq \delta d_{0} \mathrm{E} \sup _{|t| \leq \delta}\left|\left(f_{z}(W+t) g(W+t)\right)^{\prime}\right| .
\end{aligned}
$$

We first prove (4.6). By Lemma 4.7, $\left\|f_{z}\right\| \leq 1 / c_{1}$ and $\left\|f_{z}^{\prime}\right\| \leq 2$. Thus, for $0<\delta \leq 1$

$$
\begin{aligned}
& \mathrm{E}\left(\sup _{|t| \leq \delta}\left|\left(f_{z}(W+t) g(W+t)\right)^{\prime}\right|\right) \\
& \quad \leq \mathrm{E}\left(\sup _{|t| \leq \delta}\left(\left|f_{z}(W+t) g^{\prime}(W+t)\right|+\left|f_{z}^{\prime}(W+t) g(W+t)\right|\right)\right) \\
& \quad \leq\left(2+1 / c_{1}\right) \mathrm{E}\left(\sup _{|t| \leq \delta}\left(\left|g^{\prime}(W+t)\right|+|g(W+t)|\right)\right) \\
& \quad \leq 4 c_{3}\left(1+1 / c_{1}\right)\left(1+c_{2}\right)\left(\mathrm{E}|g(W)|+\mu_{1}\right)
\end{aligned}
$$

where in the last inequality we use (2.6) and Lemma 4.2. This proves (4.6) by (4.32), (4.65) and (4.66).

Next, we prove (4.7). Similar to the proof of (4.6), we first calculate the following term:

$$
\mathrm{E}\left(\sup _{|t| \leq \delta}\left|\left(f_{z}(W+t) g(W+t)\right)^{\prime}\right|\right)
$$

Note that

$$
\begin{aligned}
& \left(f_{z}(w) g(w)\right)^{\prime} \\
& = \begin{cases}\frac{p(w) g(w)+F(w) g^{\prime}(w)+F(w) g^{2}(w)}{p(w)}(1-F(z)), & w \leq z, \\
\frac{-p(w) g(w)+(1-F(w)) g^{\prime}(w)+(1-F(w)) g^{2}(w)}{p(w)} F(z), & w>z .\end{cases}
\end{aligned}
$$

For $w+t \leq 0$, by (4.2), we have

$$
\begin{aligned}
& \left|\left(f_{z}(w+t) g(w+t)\right)^{\prime}\right| \\
& \quad \leq(1-F(z))\left(2|g(w+t)|+\frac{g^{\prime}(w+t)}{\left.\max \left\{c_{1},|g(w+t)|\right\}\right)}\right) \\
& \quad \leq(1-F(z))\left(2|g(w+t)|+c_{3}\left(1+1 / c_{1}\right)\right) \\
& \quad \leq C(1-F(z))(|g(w)|+1) .
\end{aligned}
$$

imsart-generic ver. 2020/08/06 file: AAP1589-final.tex date: April 28, 2021 
Q.M. Shao, M.C. Zhang and Z.S. Zhang/Moderate deviation for nonnormal approximatiß̋

Thus, by (4.32),

$$
\mathrm{E}\left(\sup _{|t| \leq \delta}\left|\left(f_{z}(W+t) g(W+t)\right)^{\prime}\right| I(W+t \leq 0)\right) \leq C(1-F(z))
$$

For $w+t>z$, and $|t| \leq \delta$, again by Lemma 4.2, we have

$$
\begin{aligned}
& \left|\left(f_{z}(w+t) g(w+t)\right)^{\prime}\right| \\
& \quad \leq F(z)\left(|g(w+t)|+\frac{1-F(w+t)}{p(w+t)}\left(\left|g^{\prime}(w+t)\right|+|g(w+t)|^{2}\right)\right) \\
& \quad \leq C(1+|g(w+t)|) \\
& \quad \leq C(|g(w)|+1) .
\end{aligned}
$$

Hence, by Lemmas 4.5 and 4.6, we have

$$
\begin{aligned}
& \mathrm{E} \sup _{|t| \leq \delta}\left|\left(f_{z}(W+t) g(W+t)\right)^{\prime}\right| I(W+t \geq z) \\
& \quad \leq C \mathrm{E}((|g(W)|+1) I(W>z-\delta)) \\
& \quad \leq C p(z-\delta) \mathrm{E}\left(e^{G(W)-G(W-z+\delta)}|g(W)| I(W>z-\delta)\right) \\
& \quad \leq C e^{\delta g(z)} p(z)(1+g(z)) \mathrm{E}\left(e^{G(W)-G(W-z+\delta)} I(W>z-\delta)\right) \\
& \quad \leq C e^{\delta g(z)}\left(1+z g^{2}(z)\right)(1-F(z))
\end{aligned}
$$

where we use the Lemma 4.1 in the last line. Also note that by $(4.17), \delta g(z) \leq$ $\delta+\delta z g^{2}(z) \leq 2$ for $z \geq 1$ and $\delta g(z) \leq \mu_{1}$ for $0 \leq z \leq 1$. Hence,

$$
\delta g(z) \leq \max \left(2, \mu_{1}\right)
$$

Thus, (4.70) and (4.71) yield

$$
\begin{aligned}
& \mathrm{E}\left(\sup _{|t| \leq \delta}\left|\left(f_{z}(W+t) g(W+t)\right)^{\prime}\right| I(W+t>z)\right) \\
& \leq C\left(1+z g^{2}(z)\right)(1-F(z)) .
\end{aligned}
$$

For $w+t \in(0, z)$ and $|t| \leq \delta$, by (4.22), (4.67) and (4.71), we have

$$
\begin{aligned}
& \left|\left(f_{z}(w+t) g(w+t)\right)^{\prime}\right| \\
& \quad \leq C(1-F(z)) e^{G(w+t)}\left(1+g(w+t)^{2}\right) \\
& \quad \leq C(1-F(z)) e^{G(w)+\delta g(z)}\left(1+|g(w)|^{2}\right) \\
& \quad \leq C(1-F(z)) e^{G(w)}\left(1+|g(w)|^{2}\right) .
\end{aligned}
$$


Q.M. Shao, M.C. Zhang and Z.S. Zhang/Moderate deviation for nonnormal approximatißn

By Lemmas 4.5 and 4.6 and (4.22), we have

$$
\begin{aligned}
\mathrm{E}\left(\sup _{|t| \leq \delta}\left|\left(f_{z}(W+t) g(W+t)\right)^{\prime}\right| I(0 \leq W+t \leq z)\right) \\
\leq C(1-F(z)) \mathrm{E} e^{G(W)}\left(1+|g(W)|^{2}\right) I(-\delta \leq W \leq z+\delta) \\
=C(1-F(z)) \mathrm{E} e^{G(W)}\left(1+|g(W)|^{2}\right) I(-\delta \leq W \leq 0) \\
\quad+C(1-F(z)) \mathrm{E} e^{G(W)}\left(1+|g(W)|^{2}\right) I(0 \leq W \leq z+\delta) \\
\leq C e^{\mu_{1}}\left(1+\mu_{1}^{2}\right)(1-F(z)) \\
\quad+C(1-F(z))\left(1+(z+\delta) g^{2}(z+\delta)\right) \\
\leq C(1-F(z))\left(1+z g^{2}(z)\right) .
\end{aligned}
$$

Putting together (4.68), (4.72) and (4.74) gives

$$
\mathrm{E}\left(\sup _{|t| \leq \delta}\left|\left(f_{z}(W+t) g(W+t)\right)^{\prime}\right|\right) \leq C\left(1+z g^{2}(z)\right)(1-F(z)) .
$$

By (4.65) and (4.75), we obtain (4.7).

Proof of Proposition 4.2. By Lemma 4.7, we have $\left\|f_{z} g\right\| \leq 1$; thus, by (2.7) and (4.32),

$$
I_{2}+I_{3} \leq C \mathrm{E}\left|\mathrm{E}\left(\hat{K}_{1} \mid W\right)-1\right| \leq C \delta_{1}\left(\mathrm{E}\left(|g(W)|^{\tau_{1}}\right)+1\right) \leq C \delta_{1} .
$$

To bound $I_{4}$, by (2.8), (4.32) and (4.58), we have

$$
I_{4} \leq C \delta_{2}
$$

This proves (4.8).

We now move to prove (4.9) and (4.10). As to $I_{2}$, by (2.7) and Lemma 4.8, for $z \geq 0, \max \left(\delta, \delta_{1}, \delta_{2}\right) \leq 1$ and $\delta z g^{2}(z)+\delta_{1} z g^{\tau_{1}+1}(z)+\delta_{2} z g^{\tau_{2}}(z) \leq 1$, we have

$$
\begin{aligned}
I_{2} & \leq \delta_{1} \mathrm{E}\left(f_{z}(W)|g(W)|\left(|g(W)|^{\tau_{1}}+1\right)\right. \\
& \leq C \delta_{1} \mathrm{E}\left(f_{z}(W)\left(1+|g(W)|^{\tau_{1}+1}\right)\right) \\
& \leq C \delta_{1}\left(1+z g^{\tau_{1}+1}(z)\right)(1-F(z)) .
\end{aligned}
$$

As to $I_{3}$, note that

$$
I(W>z) \leq \frac{e^{G(W)-G(W-z)}}{e^{G(z)}} I(W>z) .
$$


Q.M. Shao, M.C. Zhang and Z.S. Zhang/Moderate deviation for nonnormal approximatiß2

By Lemmas 4.5 and 4.6,

$$
\begin{aligned}
& \mathrm{E}\left(\left(1+|g(W)|^{\tau_{1}}\right) I(W>z)\right) \\
& \quad \leq C p(z) \mathrm{E}\left(e^{G(W)-G(W-z)}\left(1+|g(W)|^{\tau_{1}}\right) I(W>z)\right) \\
& \quad \leq C\left(1+z g^{\tau_{1}}(z)\right) p(z) \\
& \quad \leq C\left(1+z g^{\tau_{1}+1}(z)\right)(1-F(z)),
\end{aligned}
$$

where we use (4.1) in the last inequality. Thus, by Lemma 4.5 and (4.77),

$$
\begin{aligned}
I_{3} \leq & \delta_{1}(1-F(z)) E\left(|g(W)|^{\tau_{1}}+1\right) \\
& +\delta_{1} \mathrm{E}\left(\left(|g(W)|^{\tau_{1}}+1\right) I(W>z+\delta)\right) \\
\leq & \delta_{1}(1-F(z)) E\left(|g(W)|^{\tau_{1}}+1\right) \\
& +\delta_{1} \mathrm{E}\left(\left(|g(W)|^{\tau_{1}}+1\right) I(W>z)\right) \\
\leq & C \delta_{1}\left(1+z g^{\tau_{1}+1}(z)\right)(1-F(z)) .
\end{aligned}
$$

(4.9) now follows by (4.76) and (4.78).

As to $I_{4}$, because $|R(W)| \leq \delta_{2}\left(1+|g(W)|^{\tau_{2}}\right)$, by (4.61), we have

$$
I_{4} \leq C \delta_{2}\left(1+z g^{\tau_{2}}(z)\right)(1-F(z))
$$

This completes the proof of Proposition 4.2.

\subsection{Proof of Remark 2.1}

In this subsection, we assume that the condition (2.7) in Theorem 2.1 is replaced by (2.17)-(2.19), then the result of Remark 2.1 follows from the proof of Theorem 2.1, Propositions 4.1 and 4.2 and the following proposition:

Proposition 4.3. Assume that the condition (2.7) in Theorem 2.1 is replaced by (2.17)-(2.19), then (4.8) and (4.9) hold.

Proof of Proposition 4.3. Following the proof of Proposition 4.2, it suffices to prove the following inequalities:

$$
\mathrm{E}\left|K_{2}\right| \leq \delta_{1},
$$

and for $z>0$ such that $\delta z g^{2}(z)+\delta_{1} z g^{\tau_{1}+1}(z)+\delta_{2} z g^{\tau_{2}}(z) \leq 1$,

$$
\begin{gathered}
\mathrm{E}\left|f_{z}(W) g(W) K_{2}\right| \leq C \delta_{1}\left(1+z g^{\tau_{1}+1}(z)\right)(1-F(z)), \\
\mathrm{E}\left|K_{2}\right| I(W>z) \leq C \delta_{1}\left(1+z g^{\tau_{1}+1}(z)\right)(1-F(z)) .
\end{gathered}
$$


Q.M. Shao, M.C. Zhang and Z.S. Zhang/Moderate deviation for nonnormal approximatiß3

For (4.80), by (2.19) with $s=0$, noting that $\zeta(W, 0) \equiv 1$ and $g(0)=0$, we have (4.80) holds.

For (4.81), by the definition of $f_{z}$, and by Lemmas 4.1 and 4.7 , we have

$$
\mathrm{E}\left|f_{z}(W) g(W) K_{2}\right| \leq T_{7}+T_{8}+T_{9}
$$

where

$$
\begin{aligned}
& T_{7}=(1-F(z)) \mathrm{E}\left|K_{2}\right| I(W<0), \\
& T_{8}=(1-F(z)) \mathrm{E}\left|K_{2}\right| g(W) e^{G(W)} I(0 \leq W \leq z), \\
& T_{9}=\mathrm{E}\left|K_{2}\right| I(W>z) .
\end{aligned}
$$

For $T_{7}$, by (4.80), we have

$$
T_{7} \leq \delta_{1}(1-F(z)) .
$$

For $T_{8}$, by the monotonicity of $g(\cdot)$ and by $(2.19)$ and Remark 4.1, we have

$$
\begin{aligned}
T_{8} & \leq(1-F(z)) g(z) \mathrm{E}\left|K_{2}\right| \zeta(W, z) \\
& \leq \delta_{1}(1-F(z))\left(1+g(z)^{\tau_{1}+1}\right) \mathrm{E} \zeta(W, s) \\
& \leq C \delta_{1}\left(1+z g^{\tau_{1}+1}(z)\right)(1-F(z)) .
\end{aligned}
$$

For $T_{9}$, by the Chebyshev inequality, by (2.19) and Lemmas 4.1 and 4.6 , we have

$$
\begin{aligned}
T_{9} & \leq e^{-G(z)} \mathrm{E}\left|K_{2}\right| \zeta(W, z) I(W>z) \\
& \leq C \delta_{1}\left(1+z g^{\tau_{1}}(z)\right) e^{-G(z)} \\
& \leq C \delta_{1}\left(1+z g^{\tau_{1}+1}(z)\right)(1-F(z)) .
\end{aligned}
$$

The inequality (4.81) follows from (4.83)-(4.86) while (4.82) follows from (4.86). This completes the proof.

\subsection{Proof of Remark 2.2}

In this subsection, we assume that the condition (2.11) is replaced by (2.20) and (2.21). The conclusion of Remark 2.2 follows from the proof of Theorem 2.1 and the following lemma.

Lemma 4.9. Let the conditions in Remark 2.2 be satisfied. Furthermore, $0<$ $\delta \leq 1$, and $s>0$ such that $\delta s g^{2}(s) \leq 1$. For $0 \leq \tau \leq \max \left\{2, \tau_{1}+1, \tau_{2}\right\}$, inequalities (4.32)-(4.34) hold.

imsart-generic ver. 2020/08/06 file: AAP1589-final.tex date: April 28, 2021 
Q.M. Shao, M.C. Zhang and Z.S. Zhang/Moderate deviation for nonnormal approximatiß34

Proof. Recall that $s_{0}=\max \left\{s: \delta s g^{2}(s) \leq 1\right\}$ and $\delta \leq 1$. We have

$$
s_{0} \geq s_{1} \quad \text { and } \quad \delta s_{1} g^{2}\left(s_{1}\right)=1 .
$$

Following the proof of Lemma 4.5, it suffices to prove the following two inequalities.

For $Q_{2}$ defined in (4.35),

$$
Q_{2} \leq\left(\alpha+\frac{1-\alpha}{4}\right) \mathrm{E} g_{+}^{\tau}(W)+C
$$

and for $M_{4}$ defined in (4.40),

$$
M_{4} \leq\left(\alpha+\frac{1-\alpha}{4}\right) \mathrm{E}|g(W)|^{\tau} f(W)+\left(\frac{4 \alpha}{1-\alpha}\right)^{\tau-1} \mathrm{E} f(W)+C .
$$

For $Q_{2}$, by (2.20) and similar to (4.38), we have

$$
Q_{2} \leq\left(\alpha+\frac{1-\alpha}{4}\right) \mathrm{E} g_{+}^{\tau}(W)+\left(\frac{4}{1-\alpha}\right)^{\tau-1}+d_{2} \mathrm{E} g_{+}^{\tau}(W) I(W>\kappa) .
$$

For the last term, by (2.10) and (2.21) and noting that $0 \leq \tau \leq \max \left\{2, \tau_{1}+1, \tau_{2}\right\}$, we obtain

$$
\begin{aligned}
d_{2} \mathrm{E} g_{+}^{\tau}(W) I(W>\kappa) & \leq d_{1}^{-\tau} d_{2} \delta^{-\tau} \mathrm{P}(W>\kappa) \\
& \leq d_{1}^{-\tau} d_{3} \delta^{-\tau} \exp \left(-2 s_{0} d_{1}^{-1} \delta^{-1}\right) \\
& \leq d_{1}^{-\tau} d_{3} \sup _{\delta>0}\left\{\delta^{-\tau} \exp \left(-2 s_{1} d_{1}^{-1} \delta^{-1}\right)\right\} \\
& =d_{3}\left(\frac{\tau}{2 s_{1}}\right)^{\tau} e^{-\tau}
\end{aligned}
$$

where the equality holds when $\delta=2 s_{1} /\left(d_{1} \tau\right)$. The inequality (4.87) follows from (4.38) and (4.89).

As to $M_{4}$, by $(2.20)$, we have

$$
\begin{gathered}
M_{4} \leq\left(\alpha+\frac{1-\alpha}{4}\right) \mathrm{E}|g(W)|^{\tau} f(W)+\left(\frac{4 \alpha}{1-\alpha}\right)^{\tau-1} \mathrm{E} f(W) \\
+d_{2} \mathrm{E}\left|g^{\tau}(W)\right| e^{G(W)-G(W-s)} I(W>\kappa) .
\end{gathered}
$$

For the last term, by $(2.10)$ and $(2.21)$ and noting that $g(\cdot)$ is nondecreasing 
Q.M. Shao, M.C. Zhang and Z.S. Zhang/Moderate deviation for nonnormal approximatiß5

and $s \leq s_{0}$, similar to (4.89), we have

$$
\begin{aligned}
d_{2} \mathrm{E} \mid & g^{\tau}(W) \mid e^{G(W)-G(W-s)} I(W>\kappa) \\
& \leq d_{1}^{-\tau} d_{2} \delta^{-\tau} e^{s d_{1}^{-1} \delta^{-1}} \mathrm{P}(W>\kappa) \\
& \leq d_{1}^{-\tau} d_{3} \delta^{-\tau} e^{-s_{0} d_{1}^{-1} \delta^{-1}} \\
& \leq d_{1}^{-\tau} d_{3} \sup _{\delta>0}\left\{\delta^{-\tau} e^{-s_{1} d_{1}^{-1} \delta^{-1}}\right\} \\
& =d_{3}\left(\frac{\tau}{s_{1}}\right)^{\tau} e^{-\tau}
\end{aligned}
$$

where the equality holds when $\delta=s_{1} /\left(d_{1} \tau\right)$. Combining (4.46) and (4.90), inequality (4.88) holds. Following the proof of Lemma 4.5 and replacing (4.38) and (4.46) with (4.87) and (4.88), respectively, we complete the proof of Lemma 4.9.

\section{Proofs of Theorems 3.1-3.2}

\subsection{Proof of Theorem 3.1}

In this subsection, we use Remarks 2.1 and 2.2 to prove the result.

We first prove some preliminary lemmas.

Lemma 5.1. Let $\xi \sim \rho$. For $s \in \mathbb{R}$, define

$$
\psi_{n}(s)=\frac{\mathrm{E}\left(\xi e^{\frac{\xi^{2}}{2 n}+\xi s}\right)}{\mathrm{E}\left(e^{\frac{\xi^{2}}{2 n}+\xi s}\right)}, \quad \psi_{\infty}(s)=\frac{\mathrm{E}\left(\xi e^{\xi s}\right)}{\mathrm{E}\left(e^{\xi s}\right)},
$$

and

$$
\phi_{n}(s)=\frac{\mathrm{E}\left(\xi^{2} e^{\frac{\xi^{2}}{2 n}+\xi s}\right)}{\mathrm{E}\left(e^{\frac{\xi^{2}}{2 n}+\xi s}\right)}, \quad \phi_{\infty}(s)=\frac{\mathrm{E}\left(\xi^{2} e^{\xi s}\right)}{\mathrm{E}\left(e^{\xi s}\right)}
$$

Let $m=\frac{1}{n} \sum_{i=1}^{n} X_{i}$ and $m_{i}=\frac{1}{n} \sum_{j \neq i} X_{j}$. We have for each $1 \leq i \leq n$,

$$
\begin{aligned}
& \left|\psi_{\infty}(m)-\psi_{n}\left(m_{i}\right)\right| \leq C n^{-1} \\
& \left|\phi_{\infty}(m)-\phi_{n}\left(m_{i}\right)\right| \leq C n^{-1}
\end{aligned}
$$

where $C$ is a positive constant depending only on $L$. 
Proof of Lemma 5.1. Recall that $|\xi| \leq L$ and observe that

$$
\begin{aligned}
\left|\mathrm{E}\left(\xi\left(e^{\frac{\xi^{2}}{2 n}+\xi s}-e^{\xi s}\right)\right)\right| & \leq \frac{1}{2 n} \mathrm{E}|\xi|^{3} e^{\frac{\xi^{2}}{2 n}+\xi s} \leq \frac{L^{3}}{2 n} e^{L^{2} / 2} \mathrm{E}^{\xi s}, \\
\left|\mathrm{E}\left(e^{\frac{\xi^{2}}{2 n}+\xi s}-e^{\xi s}\right)\right| & \leq \frac{1}{2 n} \mathrm{E}|\xi|^{2} e^{\frac{\xi^{2}}{2 n}+\xi s} \leq \frac{L^{2}}{2 n} e^{L^{2} / 2} \mathrm{E} e^{\xi s}, \\
\left|\mathrm{E} \xi e^{\xi s}\right| & \leq L \mathrm{E} e^{\xi s},
\end{aligned}
$$

and

$$
\mathrm{E}\left(e^{\frac{\xi^{2}}{2 n}+\xi s}\right) \geq \mathrm{E} e^{\xi s}
$$

Hence,

$$
\begin{aligned}
\left|\psi_{n}(s)-\psi_{\infty}(s)\right| \leq & \frac{\left|\mathrm{E} e^{\xi s}\right| \times\left|\mathrm{E} \xi e^{\frac{\xi^{2}}{2 n}+\xi s}-\mathrm{E} \xi e^{\xi s}\right|}{\mathrm{E} e^{\frac{\xi^{2}}{2 n}+\xi s} \mathrm{E} e^{\xi s}} \\
& +\frac{\left|\mathrm{E} \xi e^{\xi s}\right| \times\left|\mathrm{E} e^{\frac{\xi^{2}}{2 n}+\xi s}-\mathrm{E} e^{\xi s}\right|}{\mathrm{E} e^{\frac{\xi^{2}}{2 n}+\xi s} \mathrm{E} e^{\xi s}} \\
\leq & C n^{-1},
\end{aligned}
$$

where $C>0$ depends only on $L$. Moreover,

$$
\psi_{\infty}^{\prime}(s)=\frac{\mathrm{E}\left(\xi^{2} e^{\xi s}\right)}{\mathrm{E}\left(e^{\xi s}\right)}-\left\{\frac{\mathrm{E}\left(\xi e^{\xi s}\right)}{\mathrm{E}\left(e^{\xi s}\right)}\right\}^{2} .
$$

Recalling that $|\xi| \leq L,\left|X_{i}\right| \leq L$ and $\left|m-m_{i}\right| \leq L / n$, and using the fact that

$$
\sup _{|s| \leq L}\left|\psi_{\infty}^{\prime}(s)\right| \leq L^{2}
$$

we have

$$
\left|\psi_{\infty}(m)-\psi_{\infty}\left(m_{i}\right)\right| \leq L^{3} n^{-1}
$$

Following (5.3) and (5.4), the inequality (5.1) holds.

A similar argument implies that (5.2) holds as well.

Set

$$
\mathcal{F}=\sigma\left\{X_{1}, \ldots, X_{n}\right\}
$$

For any $1 \leq i, j \leq n$, define

$$
\mathcal{F}^{(i)}=\sigma\left(\left\{X_{k}, k \neq i\right\}\right) \quad \mathcal{F}^{(i, j)}=\sigma\left(\left\{X_{k}, k \neq i, j\right\}\right)
$$


Lemma 5.2. Let $W=n^{-1+\frac{1}{2 k}} \sum_{i=1}^{n} X_{i}, G(w)=h^{(2 k)}(0) w^{2 k} /(2 k)$ !, and

$$
\zeta(w, s)= \begin{cases}e^{G(w)-G(w-s)}, & w>s \\ e^{G(w)}, & 0 \leq w \leq s \\ 1, & w<0 .\end{cases}
$$

Suppose (2.9), (2.10), (2.20) and (2.21) are satisfied. Then, we have

$$
\mathrm{E}\left|\frac{1}{n} \sum_{i=1}^{n}\left(X_{i}^{2}-\mathrm{E}\left(X_{i}^{2} \mid \mathcal{F}^{(i)}\right)\right)\right| \zeta(W, s) \leq C n^{-1 / k}\left(1+|s|^{2}\right) \mathrm{E} \zeta(W, s),
$$

where $C$ is a positive constant depending only on $\rho$.

We are now ready to prove Theorem 3.1.

Proof of Theorem 3.1. We first construct the exchangeable pair of $W$. For each $1 \leq i \leq n$, let $X_{i}^{\prime}$ follow the conditional distribution of $X_{i}$ given $\left\{X_{j}, j \neq i\right\}$, and be conditionally independent of $X_{i}$ given $\left\{X_{j}, j \neq i\right\}$. Let $I$ be a random index uniformly distributed among $\{1,2, \ldots, n\}$, independent of all other random variables. Define $S_{n}^{\prime}=S_{n}-X_{I}+X_{I}^{\prime}$ and $W^{\prime}=n^{-\frac{1}{2 k}} S_{n}^{\prime}$. Then $\left(W, W^{\prime}\right)$ is an exchangeable pair. Let $\mathcal{F}, \mathcal{F}^{(i)}$ and $\mathcal{F}^{(i, j)}$ be defined as in (5.5) and (5.6). Let $\psi_{n}, \psi_{\infty}, \phi_{n}$ and $\phi_{\infty}$ be as defined in Lemma 5.1. We have

$$
\mathrm{E}\left(X_{i}^{\prime} \mid \mathcal{F}^{(i)}\right)=\mathrm{E}\left(X_{i} \mid \mathcal{F}^{(i)}\right)=\psi_{n}\left(m_{i}(\mathbf{X})\right)
$$

where $m_{i}(\mathbf{X})=\frac{1}{n} \sum_{j \neq i} X_{j}$.

Thus,

$$
\begin{aligned}
\mathrm{E}\left(X_{I}-X_{I}^{\prime} \mid \mathcal{F}\right) & =\frac{1}{n} \sum_{i=1}^{n} \mathrm{E}\left(X_{i}-X_{i}^{\prime} \mid \mathcal{F}\right) \\
& =m(\mathbf{X})-\frac{1}{n} \sum_{i=1}^{n} \mathrm{E}\left(X_{i}^{\prime} \mid \mathcal{F}^{(i)}\right) \\
& =m(\mathbf{X})-\frac{1}{n} \sum_{i=1}^{n} \psi_{n}\left(m_{i}(\mathbf{X})\right) \\
& =m(\mathbf{X})-\psi_{\infty}(m(\mathbf{X}))+r(\mathbf{X}) \\
& =h^{\prime}(m(\mathbf{X}))+r(\mathbf{X}),
\end{aligned}
$$

where $m(\mathbf{X})=(1 / n) \sum_{i=1}^{n} X_{i}, h$ is as defined in (3.3), and

$$
r(\mathbf{X})=\frac{1}{n} \sum_{i=1}^{n}\left\{\psi_{\infty}(m(\mathbf{X}))-\psi_{n}\left(m_{i}(\mathbf{X})\right)\right\}
$$


Q.M. Shao, M.C. Zhang and Z.S. Zhang/Moderate deviation for nonnormal approximatios

By Lemma 5.1, we have

$$
|r(\mathbf{X})| \leq C n^{-1}
$$

where $C>0$ is a constant depending only on $\rho$. As $\rho$ is symmetric, $h^{(2 k+1)}(0)=$ 0 . By the Taylor expansion, for $|w| \leq L$,

$$
\left|h^{\prime}(w)-g(w)\right| \leq C|w|^{2 k+1}
$$

where $C>0$ is a constant depending only on $L$. Therefore,

$$
\begin{aligned}
\mathrm{E}\left(W-W^{\prime} \mid \mathcal{F}\right) & =n^{-1+\frac{1}{2 k}} \mathrm{E}\left(X_{I}-X_{I}^{\prime} \mid \mathcal{F}\right) \\
& =n^{-1+\frac{1}{2 k}}\left(h^{\prime}(m(\mathbf{X}))+r(m(\mathbf{X}))\right) \\
& =\lambda(g(W)+R(W))
\end{aligned}
$$

where $\lambda=n^{-2+1 / k}$,

$$
g(w)=\frac{h^{(2 k)}(0)}{(2 k-1) !} w^{2 k-1}, \quad|R(w)| \leq C_{1} n^{-1 / k}\left(|w|^{2 k+1}+1\right),
$$

where $C_{1}>0$ depends only on $\rho$.

We now check the conditions $(2.20)$ and (2.21). As $g(w)=\frac{h^{(2 k)}(0)}{(k-1) !} w^{2 k-1}$, then

$$
|R(W)| \leq C_{1}\left(\frac{(k-1) !}{h^{(2 k)}(0)}+1\right) n^{-1 / k}\left(\left|W^{2} g(W)\right|+1\right)
$$

Moreover, recalling that $|W| \leq L n^{\frac{1}{2 k}}$, we have

$$
|R(W)| \leq C_{1}\left(n^{(2 k-1) / 2 k} L^{2 k+1}+1\right) .
$$

Set

$$
\kappa=\left(2 C_{1}\left(1+\frac{(k-1) !}{h^{(2 k)}(0)}\right)\right)^{-1 / 2} n^{\frac{1}{2 k}}
$$

where $d_{2}=C_{1}\left(n^{(2 k-1) / 2 k} L^{2 k+1}+\frac{(k-1) !}{h^{(2 k)}(0)}+2\right)$. Thus,

$$
|R(W)| \leq \frac{1}{2}(|g(W)|+1)+d_{2} I(|W| \geq \kappa)
$$

By Chatterjee and Dey [8, Propostion 6], for any $n \geq 1$ and $t \geq 0$,

$$
\mathrm{P}(|W| \geq t) \leq 2 e^{-c_{\rho} t^{2 k}}
$$


Q.M. Shao, M.C. Zhang and Z.S. Zhang/Moderate deviation for nonnormal approximatiß39

where $c_{\rho}>0$ is a constant depending only on $\rho$. Note that $\delta=L n^{-1+\frac{1}{2 k}}$ and by the definition of $g(\cdot)$, we have

$$
s_{0}=\max \left\{s: \delta s g^{2}(s) \leq 1\right\}=C_{2} n^{(2 k-1) /(2 k(4 k-1))},
$$

where $C_{2}>0$ is a constant depending on $\rho$. Moreover, there exists a constant $d_{1}>0$ depending on $\rho$ such that $\delta|g(W)| \leq d_{1}$. Then, there exist positive constants $C_{3}$ and $C_{4}$ depending on $\rho$ such that

$$
d_{2} e^{2 s_{0} d_{1}^{-1} \delta^{-1}} \mathrm{P}(|W| \geq \kappa) \leq C_{3}(n+1) \exp \left\{C_{4} n^{2(k-1) /(4 k-1)}-c_{\rho} n\right\} \leq d_{3},
$$

where $d_{3}>0$ is a constant depending on $\rho$. Thus the conditions (2.10), (2.20) and (2.21) hold.

For the conditional second moment, by Lemma 5.1, we have

$$
\begin{aligned}
\mathrm{E}\left(\left(X_{I}-X_{I}^{\prime}\right)^{2} \mid \mathcal{F}\right) \\
=\frac{1}{n} \sum_{i=1}^{n} \mathrm{E}\left(\left(X_{i}-X_{i}^{\prime}\right)^{2} \mid \mathcal{F}\right) \\
=\frac{1}{n} \sum_{i=1}^{n} X_{i}^{2}-\frac{2}{n} \sum_{i=1}^{n} X_{i} \psi_{n}\left(m_{i}(\mathbf{X})\right)+\frac{1}{n} \sum_{i=1}^{n} \phi_{n}\left(m_{i}(\mathbf{X})\right) \\
=\frac{1}{n} \sum_{i=1}^{n}\left(X_{i}^{2}-\phi_{n}\left(m_{i}(\mathbf{X})\right)\right)-2 m(\mathbf{X}) \psi_{\infty}(m(\mathbf{X})) \\
\quad+2 \phi_{\infty}(m(\mathbf{X}))+r_{2}(\mathbf{X})
\end{aligned}
$$

where $\psi_{n}, \phi_{n}$ and $\phi_{\infty}$ are as defined in Lemma 5.1. By the Taylor expansion, we have

$$
\left|\phi_{\infty}(m(\mathbf{X}))-1\right|=\left|h^{\prime \prime}(m(\mathbf{X}))\right| \leq C n^{-1+1 / k}\left(1+|W|^{2 k-2}\right),
$$

and

$$
\left|m(\mathbf{X}) \psi_{\infty}(m(\mathbf{X}))\right| \leq C n^{-1 / k}|W|^{2}+C n^{-1}|W|^{2 k},
$$

where $C>0$ is a constant depending only on $\rho$. By the definition of $\left(W, W^{\prime}\right)$ and (5.13)-(5.15), with $\lambda=n^{-2+1 / k}$, we have

$$
\begin{aligned}
\mid \frac{1}{2 \lambda} \mathrm{E} & \left(\left(W-W^{\prime}\right)^{2} \mid \mathcal{F}\right)-1 \mid \\
& =\left|\frac{1}{2} \mathrm{E}\left(\left(X_{I}-X_{I}^{\prime}\right)^{2} \mid \mathcal{F}\right)-1\right| \\
& \leq \frac{1}{2}\left|\frac{1}{n} \sum_{i=1}^{n}\left(X_{i}^{2}-\phi_{n}\left(m_{i}(\mathbf{X})\right)\right)\right|+C n^{-1 / k}\left(1+|W|^{2}\right) .
\end{aligned}
$$

imsart-generic ver. 2020/08/06 file: AAP1589-final.tex date: April 28, 2021 
Q.M. Shao, M.C. Zhang and Z.S. Zhang/Moderate deviation for nonnormal approximationo

Moreover, as $\left|X_{i}\right| \leq L$, we have

$$
\left|\frac{1}{2 \lambda} \mathrm{E}\left(\left(W-W^{\prime}\right)^{2} \mid \mathcal{F}\right)-1\right| \leq 2 L^{2}+1:=d_{0} .
$$

Then (2.9) holds. By Lemma 5.2, we have the condition (2.19) in Remark 2.1 is satisfied.

Hence, we have (2.8)-(2.10) and the conditions in Remarks 2.1 and 2.2 are satisfied with $\delta_{1}=\delta_{2}=C n^{-1 / k}, \tau_{1}=\frac{2}{2 k-1}$, and $\tau_{2}=1+\frac{2}{2 k-1}$. By Remarks 2.1 and 2.2, we complete the proof of Theorem 3.1.

It suffices to proof Lemma 5.2.

Proof of Lemma 5.2. In this proof, we denote $C$ by a general positive constant depending only on $\rho$. By the Cauchy inequality, we have

$$
\begin{aligned}
\mathrm{E} \mid \frac{1}{n} & \sum_{i=1}^{n}\left(X_{i}^{2}-\mathrm{E}\left(X_{i}^{2} \mid \mathcal{F}^{(i)}\right)\right) \mid \zeta(W, s) \\
& \leq\left(\mathrm{E}\left|\frac{1}{n} \sum_{i=1}^{n}\left(X_{i}^{2}-\mathrm{E}\left(X_{i}^{2} \mid \mathcal{F}^{(i)}\right)\right)\right|^{2} \zeta(W, s) \times \mathrm{E} \zeta(W, s)\right)^{1 / 2} .
\end{aligned}
$$

Expand the square term, and we have

$$
\mathrm{E}\left|\frac{1}{n} \sum_{i=1}^{n}\left(X_{i}^{2}-\mathrm{E}\left(X_{i}^{2} \mid \mathcal{F}^{(i)}\right)\right)\right|^{2} \zeta(W, s)=H_{1}+H_{2},
$$

where

$$
\begin{aligned}
& H_{1}=\frac{1}{n^{2}} \sum_{i=1}^{n} \mathrm{E}\left\{\left(X_{i}^{2}-\mathrm{E}\left(X_{i}^{2} \mid \mathcal{F}^{(i)}\right)\right)^{2} \zeta(W, s)\right\} \\
& H_{2}=\frac{1}{n^{2}} \sum_{i \neq j} \mathrm{E}\left\{\left(X_{i}^{2}-\mathrm{E}\left(X_{i}^{2} \mid \mathcal{F}^{(i)}\right)\right)\left(X_{j}^{2}-\mathrm{E}\left(X_{j}^{2} \mid \mathcal{F}^{(j)}\right)\right) \zeta(W, s)\right\}
\end{aligned}
$$

Recalling that $\left|X_{i}\right| \leq L$, we have

$$
H_{1} \leq 4 L^{4} n^{-1} \mathrm{E} \zeta(W, s)
$$

As for $H_{2}$, we first introduce some notations. For $i \neq j$, let $\mathrm{E}^{(i, j)}$ denote the conditional expectation given $\mathcal{F}^{(i, j)}$, where $\mathcal{F}^{(i, j)}$ is as in (5.6). Note that

$$
\mathrm{E}^{(i, j)}\left(X_{i}^{2}\right)=\frac{\iint x^{2} \exp \left(\frac{1}{2 n}(x+y)^{2}+(x+y) m_{i j}\right) \mathrm{d} \rho(x) \mathrm{d} \rho(y)}{\iint \exp \left(\frac{1}{2 n}(x+y)^{2}+(x+y) m_{i j}\right) \mathrm{d} \rho(x) \mathrm{d} \rho(y)},
$$

imsart-generic ver. 2020/08/06 file: AAP1589-final.tex date: April 28, 2021 
Q.M. Shao, M.C. Zhang and Z.S. Zhang/Moderate deviation for nonnormal approximation

where $m_{i j}:=m_{i j}(\mathbf{X})=\frac{1}{n} \sum_{k \neq i, j} X_{k}$. Similar to Lemma 5.1, we have for any $i \neq j$,

$$
\left|\mathrm{E}\left(X_{i}^{2} \mid \mathcal{F}^{(i)}\right)-\mathrm{E}^{(i, j)}\left(X_{i}^{2}\right)\right| \leq C n^{-1},
$$

where $C>0$ depends only on $L$. Define

$$
H_{3}=\frac{1}{n^{2}} \sum_{i \neq j} \mathrm{E}\left\{\left(X_{i}^{2}-\mathrm{E}^{(i, j)}\left(X_{i}^{2}\right)\right)\left(X_{j}^{2}-\mathrm{E}^{(i, j)}\left(X_{j}^{2}\right)\right) \zeta(W, s)\right\}
$$

and then by (5.19) and (5.20), we have

$$
\left|H_{2}-H_{3}\right| \leq C n^{-1} \mathrm{E} \zeta(W, s)
$$

We now move to give the bound of $H_{3}$. Define

$$
W^{(i, j)}=W-n^{-1+\frac{1}{2 k}}\left(X_{i}+X_{j}\right) .
$$

Let

$$
q(w, s)= \begin{cases}G(w)-G(w-s), & w>s \\ G(w), & 0 \leq w \leq s \\ 0, & w<0\end{cases}
$$

and then $q(w, s)=\log \zeta(w, s)$ and $q^{\prime}(w)$ is continuous on $\mathbb{R}$. Therefore, by the Taylor expansion, we have

$$
\begin{aligned}
q(W)-q\left(W^{(i, j)}\right)= & \left(W-W^{(i, j)}\right) q^{\prime}\left(W^{(i, j)}\right) \\
& +\frac{1}{2}\left(W-W^{(i, j)}\right)^{2} q^{\prime \prime}\left(w_{0}\right),
\end{aligned}
$$

where $w_{0}$ belongs to either $\left(W, W^{(i, j)}\right)$ or $\left(W^{(i, j)}, W\right)$. Note that $G(w)=C w^{2 k}$ for some constant $C,|W| \leq L n^{\frac{1}{2 k}}$ and $\left|W-W^{(i, j)}\right| \leq 2 L n^{-1+\frac{1}{2 k}}$. By the definition of $q$, we have

$$
\begin{aligned}
\mid(W & \left.-W^{(i, j)}\right) q^{\prime}\left(W^{(i, j)}\right) \mid \\
& \leq C n^{-1+\frac{1}{2 k}}\left|W^{(i, j)}\right|^{2 k-1} \\
& \leq C n^{-1+\frac{1}{2 k}}\left(|W|^{2 k-1}+1\right)
\end{aligned}
$$

and

$$
\left|\frac{1}{2}\left(W-W^{(i, j)}\right)^{2} q^{\prime \prime}\left(w_{0}\right)\right| \leq C n^{-1}
$$


Q.M. Shao, M.C. Zhang and Z.S. Zhang/Moderate deviation for nonnormal approximation

where $C$ depends only on $\rho$. Therefore, by (5.22)-(5.24) and using the fact that $|W| \leq \operatorname{Ln} \frac{1}{2 k}$, we have

$$
\begin{aligned}
\left|q(W)-q\left(W^{(i, j)}\right)\right| & \leq C n^{-1+\frac{1}{2 k}}\left(|W|^{2 k-1}+1\right) \\
& \leq C
\end{aligned}
$$

Observe that

$$
\mathrm{E}^{(i, j)}\left\{\left(X_{i}^{2}-\mathrm{E}^{(i, j)}\left(X_{i}^{2}\right)\right)\left(X_{j}^{2}-\mathrm{E}^{(i, j)}\left(X_{j}^{2}\right)\right) \zeta(W, s)\right\}=\zeta\left(W^{(i, j)}\right) M^{(i, j)}
$$

where

$$
M^{(i, j)}=\mathrm{E}^{(i, j)}\left\{\left(X_{i}^{2}-\mathrm{E}^{(i, j)}\left(X_{i}^{2}\right)\right)\left(X_{j}^{2}-\mathrm{E}^{(i, j)}\left(X_{j}^{2}\right)\right) e^{q(W)-q\left(W^{(i, j)}\right)}\right\} .
$$

Applying the Taylor expansion to the exponential function, we have

$$
M^{(i, j)}=M_{1}^{(i, j)}+M_{2}^{(i, j)}+M_{3}^{(i, j)}
$$

where

$$
\begin{aligned}
& M_{1}^{(i, j)}=\mathrm{E}^{(i, j)}\left\{\left(X_{i}^{2}-\mathrm{E}^{(i, j)} X_{i}^{2}\right)\left(X_{j}^{2}-\mathrm{E}^{(i, j)} X_{j}^{2}\right)\right\}, \\
& M_{2}^{(i, j)}=\mathrm{E}^{(i, j)}\left(\left(X_{i}^{2}-\mathrm{E}^{(i, j)} X_{i}^{2}\right)\left(X_{j}^{2}-\mathrm{E}^{(i, j)} X_{j}^{2}\right)\left\{q(W)-q\left(W^{(i, j)}\right)\right\}\right),
\end{aligned}
$$

and

$$
M_{3}^{(i, j)}=M^{(i, j)}-M_{1}^{(i, j)}-M_{2}^{(i, j)} .
$$

For $M_{1}^{(i, j)}$, since $\mathrm{E}^{(i, j)} X_{i}^{2}=\mathrm{E}^{(i, j)} X_{j}^{2}$, we have

$$
\begin{aligned}
M_{1}^{(i, j)}= & \mathrm{E}^{(i, j)} X_{i}^{2} X_{j}^{2}-\mathrm{E}^{(i, j)} X_{i}^{2} \mathrm{E}^{(i, j)} X_{j}^{2} \\
= & \frac{\iint x^{2} y^{2} \exp \left(\frac{1}{2 n}(x+y)^{2}+(x+y) m_{i j}\right) \mathrm{d} \rho(x) \mathrm{d} \rho(y)}{\iint \exp \left(\frac{1}{2 n}(x+y)^{2}+(x+y) m_{i j}\right) \mathrm{d} \rho(x) \mathrm{d} \rho(y)} \\
& \quad-\left(\frac{\iint x^{2} \exp \left(\frac{1}{2 n}(x+y)^{2}+(x+y) m_{i j}\right) \mathrm{d} \rho(x) \mathrm{d} \rho(y)}{\iint \exp \left(\frac{1}{2 n}(x+y)^{2}+(x+y) m_{i j}\right) \mathrm{d} \rho(x) \mathrm{d} \rho(y)}\right)^{2} \\
= & M_{11}^{(i, j)}+M_{12}^{(i, j)}
\end{aligned}
$$

where

$$
\begin{aligned}
M_{11}^{(i, j)}= & \frac{\iint x^{2} y^{2} \exp \left((x+y) m_{i j}\right) \mathrm{d} \rho(x) \mathrm{d} \rho(y)}{\iint \exp \left((x+y) m_{i j}\right) \mathrm{d} \rho(x) \mathrm{d} \rho(y)} \\
& \quad-\left(\frac{\iint x^{2} \exp \left((x+y) m_{i j}\right) \mathrm{d} \rho(x) \mathrm{d} \rho(y)}{\iint \exp \left((x+y) m_{i j}\right) \mathrm{d} \rho(x) \mathrm{d} \rho(y)}\right)^{2} \\
= & 0,
\end{aligned}
$$


Q.M. Shao, M.C. Zhang and Z.S. Zhang/Moderate deviation for nonnormal approximatioß and $M_{12}^{(i, j)}=M_{1}^{(i, j)}-M_{11}^{(i, j)}$. Similar to Lemma 5.1, we have

$$
\left|M_{12}^{(i, j)}\right| \leq C n^{-1}
$$

By (5.28) and (5.29), we have

$$
\left|M_{1}^{(i, j)}\right| \leq C n^{-1}
$$

For $M_{2}^{(i, j)}$, by (5.22) and (5.24), we have

$$
M_{2}^{(i, j)}=M_{21}^{(i, j)}+M_{22}^{(i, j)}
$$

where

$$
\begin{aligned}
& M_{21}^{(i, j)}=n^{-1+\frac{1}{2 k}} q^{\prime}\left(W^{(i, j)}\right) \mathrm{E}^{(i, j)}\left\{\left(X_{i}^{2}-\mathrm{E}^{(i, j)} X_{i}^{2}\right)\left(X_{j}^{2}-\mathrm{E}^{(i, j)} X_{j}^{2}\right)\left(X_{i}+X_{j}\right)\right\}, \\
& M_{22}^{(i, j)}=\frac{1}{2} \mathrm{E}^{(i, j)}\left\{\left(X_{i}^{2}-\mathrm{E}^{(i, j)} X_{i}^{2}\right)\left(X_{j}^{2}-\mathrm{E}^{(i, j)} X_{j}^{2}\right)\left(W-W^{(i, j)}\right)^{2} q^{\prime \prime}\left(w_{0}\right)\right\},
\end{aligned}
$$

and $w_{0}$ is as defined in (5.22). By (5.24), and recalling that $\left|X_{i}\right| \leq L$, we have

$$
\left|M_{22}^{(i, j)}\right| \leq C n^{-1} .
$$

Similar to (5.30), we have

$$
\left|\mathrm{E}^{(i, j)}\left\{\left(X_{i}^{2}-\mathrm{E}^{(i, j)} X_{i}^{2}\right)\left(X_{j}^{2}-\mathrm{E}^{(i, j)} X_{j}^{2}\right)\left(X_{i}+X_{j}\right)\right\}\right| \leq C n^{-1} .
$$

Moreover, recalling that $\left|W^{(i, j)}\right| \leq L n^{\frac{1}{2 k}}$ and $\left|q^{\prime}\left(W^{(i, j)}\right)\right| \leq C n^{1-\frac{1}{2 k}}$, we have

$$
\left|M_{21}^{(i, j)}\right| \leq C n^{-1} \text {. }
$$

Thus,

$$
\left|M_{2}^{(i, j)}\right| \leq C n^{-1} .
$$

For $M_{3}^{(i, j)}$, by the Taylor expansion, noting again that $k \geq 2,|W| \leq L n^{\frac{1}{2 k}}$ and $\left|X_{i}\right| \leq L$ for $1 \leq i \leq n$, and by (5.23) and (5.24), we have

$$
\begin{aligned}
\left|M_{3}^{(i, j)}\right| & \leq C\left|q(W)-q\left(W^{(i, j)}\right)\right|^{2} e^{q(W)-q\left(W^{(i, j)}\right)} \\
& \leq C n^{-2+1 / k}\left(|W|^{4 k-2}+1\right) \\
& \leq C n^{-2 / k}\left(|W|^{4}+1\right) .
\end{aligned}
$$

By (5.27) and (5.30)-(5.32), we have

$$
\left|M^{(i, j)}\right| \leq C n^{-2 / k}\left(|W|^{4}+1\right),
$$


Q.M. Shao, M.C. Zhang and Z.S. Zhang/Moderate deviation for nonnormal approximatiom

substituting which to (5.26), we have

$$
\begin{aligned}
& \mathrm{E}\left|\mathrm{E}^{(i, j)}\left\{\left(X_{i}^{2}-\mathrm{E}^{(i, j)}\left(X_{i}^{2}\right)\right)\left(X_{j}^{2}-\mathrm{E}^{(i, j)}\left(X_{j}^{2}\right)\right) \zeta(W, s)\right\}\right| \\
& \leq C n^{-2 / k} \mathrm{E}\left\{\left(|W|^{4}+1\right) \zeta\left(W^{(i, j)}\right)\right\} \\
& \leq C n^{-2 / k} \mathrm{E}\left\{\left(|W|^{4}+1\right) \zeta(W, s)\right\} \\
& \leq C n^{-2 / k}\left(1+s^{4}\right) \mathrm{E} \zeta(W, s),
\end{aligned}
$$

where in the last inequality we used Lemma 4.9 recalling the fact that (5.11) and (5.12) are satisfied. By (5.33), we have the term $H_{3}$ in (5.20) can be bounded by

$$
\left|H_{3}\right| \leq C n^{-2 / k}\left(1+s^{4}\right) \mathrm{E} \zeta(W, s) .
$$

By (5.16)-(5.18), (5.21) and (5.34), we complete the proof of (5.7).

\subsection{Proof of Theorem 3.2}

In this subsection, we use Remark 2.2 to prove the result.

Proof of Theorem 3.2. For any $\sigma \in \Sigma, u v \in D$ and $s, t \in\{0,1\}$, let $\sigma_{u v}^{s t}$ denote the configuration $\tau \in \Sigma$, such that $\tau_{i}=\sigma_{i}$ for $i \neq u, v$ and $\tau_{u}=s, \tau_{v}=t$. Let $\left(\sigma_{u}^{\prime}, \sigma_{v}^{\prime}\right)$ be independent of $\left(\sigma_{u}, \sigma_{v}\right)$ and follow the conditional distribution

$$
\mathrm{P}\left(\sigma_{u}^{\prime}=s, \sigma_{v}^{\prime}=t \mid \sigma\right)=\frac{p\left(\sigma_{u v}^{s t}\right)}{\sum_{s, t \in\{0,1\}} p\left(\sigma_{u v}^{s t}\right)} .
$$

Let $M=\sum_{i=1}^{n} \sigma_{i}$ and $M^{\prime}=M-\sigma_{u}-\sigma_{v}+\sigma_{u}^{\prime}+\sigma_{v}^{\prime}$. Then, by Chen [15], $\left(M, M^{\prime}\right)$ is exchangeable. Also, by Chen [15, Proposition 2], we have

$$
\begin{aligned}
\mathrm{E}\left(M-M^{\prime} \mid \sigma\right) & =L_{1}(m(\sigma))+R_{1}(m(\sigma)), \\
\mathrm{E}\left(\left(M-M^{\prime}\right)^{2} \mid \sigma\right) & =L_{2}(m(\sigma))+R_{2}(m(\sigma)),
\end{aligned}
$$

where $m(\sigma)=M / n$ and

$$
\begin{gathered}
L_{1}(x)=\frac{2(1-x)\left(x^{2}-(1-x) e^{2 \tau(x)}\right)}{(1-x)+e^{2 \tau(x)}}, \text { for } 0<x<1, \\
L_{2}(x)=\frac{4(1-x)\left(x^{2}+(1-x) e^{2 \tau(x)}\right)}{(1-x)+e^{2 \tau(x)}}, \text { for } 0<x<1, \\
\left|R_{1}(x)\right|+\left|R_{2}(x)\right| \leq \frac{C}{n}
\end{gathered}
$$

for some constant $C$. Next, we consider two cases. In the first case, $(J, h) \notin$ $\Gamma \cup\left\{\left(J_{c}, h_{c}\right)\right\}$, and in the second case, $(J, h)=\left(J_{c}, h_{c}\right)$. 
Q.M. Shao, M.C. Zhang and Z.S. Zhang/Moderate deviation for nonnormal approximatiołs

Case 1. When $(J, h) \notin \Gamma \cup\left\{\left(J_{c}, h_{c}\right)\right\}$. Define $W=n^{-1 / 2}\left(M-n m_{0}\right)$ and $W^{\prime}=n^{-1 / 2}\left(M^{\prime}-n m_{0}\right)$; then, $\left(W, W^{\prime}\right)$ is also an exchangeable pair. Moreover,

$$
\left|W-W^{\prime}\right| \leq 2 n^{-1 / 2}=: \delta .
$$

Note that $L_{1}\left(m_{0}\right)=0$ by observing $m_{0}^{2}=\left(1-m_{0}\right) e^{2 \tau\left(m_{0}\right)}$. Moreover, we have

$$
L_{1}^{\prime}\left(m_{0}\right)=\frac{1}{2 \lambda_{0}} L_{2}\left(m_{0}\right)>0
$$

where $\lambda_{0}=\left(-1 / H^{\prime \prime}\left(m_{0}\right)\right)-(1 / 2 J)>0$. By the Taylor expansion, we have

$$
L_{1}(m(\sigma))=L_{1}^{\prime}\left(m_{0}\right)\left(m(\sigma)-m_{0}\right)+\int_{m_{0}}^{m(\sigma)} L_{1}^{\prime \prime}(s)(m(\sigma)-s) d s .
$$

Let $\lambda=L_{2}\left(m_{0}\right) /(2 n)$, and we have

$$
n^{-1 / 2} L_{1}(m(\sigma))=\lambda\left(\lambda_{0}^{-1} W+r(W)\right),
$$

where

$$
r(W)=2 n^{1 / 2} L_{2}^{-1}\left(m_{0}\right) \int_{m_{0}}^{m(\sigma)} L_{1}^{\prime \prime}(s)(m(\sigma)-s) d s .
$$

Therefore, together with the definition of $\left(W, W^{\prime}\right)$ and $(5.35)$, we have

$$
\mathrm{E}\left(W-W^{\prime} \mid W\right)=n^{-1 / 2}\left(L_{1}(m(\sigma))+R_{1}(m(\sigma))\right)=\lambda(g(W)+R(W)),
$$

where

$$
g(W)=W / \lambda_{0} \quad \text { and } \quad R(W)=r(W)+\frac{2 n^{1 / 2}}{L_{2}\left(m_{0}\right)} R_{1}(m(\sigma)) .
$$

Thus, conditions (A1)-(A4) hold for $g(w)=w / \lambda_{0}$. Furthermore, $\delta|g(W)| \leq$ $2 / \lambda_{0}$, as $n^{-1 / 2}|W| \leq 1$.

By Chen [15, Lemma 1], there exist constants $C_{0}, C_{1}>0$ such that

$$
|R(W)| \leq C_{0} n^{-1 / 2}\left(W^{2}+1\right),
$$

and

$$
\left|\frac{1}{2 \lambda} \mathrm{E}\left(\left(W-W^{\prime}\right)^{2} \mid W\right)-1\right| \leq C_{1} n^{-1 / 2}(|W|+1)
$$

and $\left|\hat{K}_{1}\right|=\frac{\Delta^{2}}{2 \lambda} \leq 4 / L_{2}\left(m_{0}\right)$. Therefore, (2.7)-(2.10) are satisfied with $\tau_{1}=$ $1, \tau_{2}=2, \delta_{1}=\delta_{2}=O(1) n^{-1 / 2}$ and $d_{0}=4 / L_{2}\left(m_{0}\right)$ and $d_{1}=2 / \lambda_{0}$.

It suffices to prove (2.20) and (2.21). By (5.37), we have for $|W| \leq \frac{\sqrt{n}}{2 \lambda_{0} C_{0}}$,

$$
|R(W)| \leq \frac{1}{2}(|g(W)|+1)
$$


Q.M. Shao, M.C. Zhang and Z.S. Zhang/Moderate deviation for nonnormal approximation

and for $|W|>\frac{\sqrt{n}}{2 \lambda_{0} C_{0}}$, recalling that $|W| \leq 1$, we have $|R(W)| \leq C_{0}(\sqrt{n}+1)$. Then, (2.20) holds with $\alpha=1 / 2, d_{2}=C_{0}(\sqrt{n}+1)$ and $\kappa=\sqrt{n} /\left(2 \lambda_{0} C_{0}\right)$. By Chen [15, Lemma 2], when $(J, h) \notin \Gamma \cup\left\{\left(J_{c}, h_{c}\right)\right\}$, for any $u>0$, there exists a constant $\eta>0$ such that

$$
\mathrm{P}\left(\left|m(\sigma)-m_{0}\right| \geq u\right) \leq C e^{-n \eta}
$$

for some constant $C$. Hence,

$$
d_{2} \mathrm{P}(|W|>\kappa) \leq C(\sqrt{n}+1) e^{-n \eta} .
$$

Note that $s_{0}=\max \left\{s: \delta s g^{2}(s) \leq 1\right\}, g(w)=w / \lambda_{0}, d_{1}=\frac{2}{\lambda_{0}}$ and $\delta=2 n^{-1 / 2}$, then $s_{0}=\left(\lambda_{0} / 2\right)^{1 / 3} n^{1 / 6}$. Therefore, (2.21) is satisfied. By Remark 2.2, we have

$$
\frac{\mathrm{P}(W>z)}{\mathrm{P}\left(Z_{0}>z\right)}=1+O(1) n^{-1 / 2}\left(1+z^{3}\right)
$$

for $0 \leq z \leq n^{1 / 6}$.

Case 2. When $(J, h)=\left(J_{c}, h_{c}\right)$. Define $W=n^{-3 / 4}\left(M-n m_{c}\right)$ and $W^{\prime}=$ $n^{-3 / 4}\left(M^{\prime}-n m_{c}\right)$; then, $\left(W, W^{\prime}\right)$ is an exchangeable pair. By (5.35), we have

$$
\mathrm{E}\left(W-W^{\prime} \mid W\right)=n^{-3 / 4}\left(L_{1}(m(\sigma))+R_{1}(m(\sigma))\right) .
$$

By Chen [15, p. 14], we have

$$
L_{1}\left(m_{c}\right)=L_{1}^{\prime}\left(m_{c}\right)=L_{1}^{\prime \prime}\left(m_{c}\right)=0, \quad L_{1}^{(3)}\left(m_{c}\right)=\frac{\lambda_{c}}{2} L_{2}\left(m_{c}\right),
$$

where $\lambda_{c}$ is given in (3.8). Then, by the Taylor expansion, we have

$$
L_{1}(m(\sigma))=\frac{L_{1}^{(3)}\left(m_{c}\right)}{6}\left(m(\sigma)-m_{c}\right)^{3}+\frac{1}{6} \int_{m_{c}}^{m(\sigma)} L_{1}^{(4)}(s)(m(\sigma)-s)^{3} d s .
$$

Then, taking $\lambda=L_{2}\left(m_{c}\right) /\left(2 n^{3 / 2}\right)$, by Chen [15, Lemma 1], we have

$$
\mathrm{E}\left(W-W^{\prime} \mid W\right)=\lambda(g(W)+R(W))
$$

where $g(W)=\left(\lambda_{c} / 6\right) W^{3}$ and

$$
R(W)=\frac{n^{3 / 4}}{2 L_{2}\left(m_{c}\right)} \int_{m_{0}}^{m(\sigma)} L_{1}^{(4)}(s)\left(m(\sigma)-m_{c}\right)^{3} d s+\frac{2 n^{3 / 4}}{L_{2}\left(m_{c}\right)} R_{1}(W) .
$$

Hence, $G(w)=\frac{\lambda_{c}}{24} w^{4}$. Based again on Chen [15, Lemma 1], for some constant $C$, we have

$$
|R(W)| \leq C n^{-1 / 4}\left(|W|^{4}+1\right) \leq C n^{-1 / 4}\left(|g(W)|^{4 / 3}+1\right),
$$


Q.M. Shao, M.C. Zhang and Z.S. Zhang/Moderate deviation for nonnormal approximation

and

$$
\left|\frac{1}{2 \lambda} \mathrm{E}\left(\left(W-W^{\prime}\right)^{2} \mid W\right)-1\right| \leq C n^{-1 / 4}\left(|g(W)|^{1 / 3}+1\right) .
$$

As $\left|W-W^{\prime}\right| \leq 2 n^{-3 / 4}$ and $|W| \leq C n^{1 / 4}$, it follows that there exist constants $d_{0}$ and $d_{1}$ such that $n^{-3 / 4}|g(W)| \leq d_{1}$ and $\hat{K}_{1}=\left(W-W^{\prime}\right)^{2} /(2 \lambda) \leq d_{0}$. Thus, (2.9) and (2.10) are satisfied. Furthermore, (2.7) and (2.8) hold with $\delta=2 n^{-3 / 4}, \delta_{1}=$ $\delta_{2}=O(1) n^{-1 / 4}$ and $\tau_{1}=1 / 3, \tau_{2}=4 / 3$. It suffices to show that $(2.20)$ and $(2.21)$ are satisfied. By (5.39), there exists a constant $c>0$ such that for $|W| \leq c n^{1 / 4}$,

$$
|R(W)| \leq \frac{1}{2}(|g(W)|+1) .
$$

For $|W| \geq c n^{1 / 4}$, noting that $|W| \leq C n^{1 / 4}$, we have $|R(W)| \leq C n^{3 / 4}$. Thus, (2.20) is satisfied with $\alpha=1 / 2, d_{2}=C n^{3 / 4}$ and $\kappa=c n^{1 / 4}$. Furthermore, as $\delta=2 n^{-3 / 4}$ and $g(w)=\left(\lambda_{c} / 6\right) w^{3}$, we have $s_{0}=\left(18 / \lambda_{c}\right)^{1 / 7} n^{3 / 28}$. In addition, by Chen [15, Lemma 2], when $(J, h)=\left(J_{c}, h_{c}\right)$, for any $u>0$, there exists a constant $\eta>0$ such that

$$
\mathrm{P}\left(\left|m(\sigma)-m_{c}\right| \geq u\right) \leq C e^{-n \eta}
$$

Thus,

$$
d_{2} \mathrm{P}(|W| \geq \kappa) \leq C n^{3 / 4} e^{-n \eta} \leq C e^{-2 s_{0} d_{1}^{-1} \delta^{-1}}
$$

Then, (2.21) holds. By Remark 2.2, we complete the proof of Theorem 3.2.

\section{Acknowledgements}

We would like to thank the referees for their helpful comments which led to a much improved presentation of the paper.

\section{References}

[1] Alberici, D., Contucci, P., Fedele, M. and Mingione, E. (2016). Limit theorems for monomer-dimer mean-field models with attractive potential. Commun. Math. Phys., 346(3), 781-799.

[2] Alberici, D., Contucci, P. and Mingione, E. (2014). A mean-field monomerdimer model with attractive interaction: Exact solution and rigorous results. J. Math. Phys., 55(6), 063301. 
Q.M. Shao, M.C. Zhang and Z.S. Zhang/Moderate deviation for nonnormal approximatiow

[3] Barbour, A. (1990). Stein's method for diffusion approximations. Probab. Theory Relat. Fields, 84(3), 297-322.

[4] Can, V. H. and Pham, V.-H. (2017). A Cramér type moderate deviation theorem for the critical Curie-Weiss model. Electron. Commun. Probab., 22(62), 1-12.

[5] Chang, T. S. (1939). Statistical theory of the adsorption of double molecules. Proceedings of the Royal Society of London. Series A, Mathematical and Physical Sciences, 169(939), 512-531.

[6] Chatterjee, S. (2008). A new method of normal approximation. Ann. Probab., 36(4), 1584-1610.

[7] Chatterjee, S. (2014). A short survey of Stein's method. Proceedings of ICM 2014., 4, 1-24.

[8] Chatterjee, S. and Dey, P. S. (2010). Applications of Stein's method for concentration inequalities. Ann. Probab., 38(6), 2443-2485.

[9] Chatterjee, S. and Shao, Q.-M. (2011). Nonnormal approximation by Stein's method of exchangeable pairs with application to the Curie-Weiss model. Ann. Probab., 21(2), 464-483.

[10] Chen, L. H. Y. and Shao, Q.-M. (2004). Normal approximation under local dependence. Ann. Probab., 32(3), 1985-2028.

[11] Chen, L. H. Y. and Shao, Q.-M. (2007). Normal approximation for nonlinear statistics using a concentration inequality approach. Bernoulli, 13(2), 581-599.

[12] Chen, L. H. Y., Fang, X. and Shao, Q.-M. (2013). From Stein identities to moderate deviations. Ann. Probab., 41(1), 262-293.

[13] Chen, L. H. Y., Goldstein, L. and Shao, Q.-M. (2011). Normal approximation by Stein's method. Probability and its Applications. Springer, Heidelberg.

[14] Chen, L. H. Y. and Röllin, A. (2010). Stein couplings for normal approximation. ArXiv: 1003.6039.

[15] Chen, W.-K. (2016). Limit theorems in the imitative monomer-dimer meanfield model via Stein's method. J. Math. Phys., 57(8), 083302.

[16] Diaconis, P. (1977). Finite forms of de Finetti's theorem on exchangeability. Synthese, 36(2), 271-281.

[17] Ellis, R. S. and Newman, C. M. (1978). The statistics of Curie-Weiss models. J. Stat. Phys., 19(2), 149-161.

imsart-generic ver. 2020/08/06 file: AAP1589-final.tex date: April 28, 2021 
Q.M. Shao, M.C. Zhang and Z.S. Zhang/Moderate deviation for nonnormal approximationg

[18] Fowler, R. H. and Rushbrooke, G. S. (1937). An attempt to extend the statistical theory of perfect solutions. Trans. Faraday Soc., 33, 1272-1294.

[19] Goldstein, L. and Reinert, G. (1997). Stein's method and the zero bias transformation with application to simple random sampling. Ann. Probab., 7(4), 935-952.

[20] Linnik, Y. V. (1961). On the probability of large deviations for the sums of independent variables. In Proceedings of the Fourth Berkeley Symposium on Mathematical Statistics and Probability, 2, 289-306. University of California Press, Berkeley.

[21] Nourdin, I. and Peccati, G. (2009). Stein's method on Wiener chaos. Probab. Theory Relat. Fields, 145(1-2), 75-118.

[22] Nourdin, I. and Peccati, G. (2012). Normal approximations with Malliavin calculus: from Stein's method to universality. Cambridge University Press, Cambridge.

[23] Petrov, V. V. (1975). Sums of independent random variables. SpringerVerlag, New York-Heidelberg, translated from the Russian by A. A. Brown, Ergebnisse der Mathematik und ihrer Grenzgebiete, Band 82.

[24] Roberts, J. K. (1938). Some properties of mobile and immobile adsorbed films. Proceedings of the Cambridge Philosophical Society, 34, p. 399.

[25] Shao, Q.-M. and Zhang, Z.-S. (2016). Identifying the limiting distribution by a general approach of Stein's method. Sci. China-Math., 59(12), 2379-2392.

[26] Shao, Q.-M. and Zhang, Z.-S. (2019). Berry-Esseen bounds of normal and nonnormal Approximation for unbounded exchangeable pairs. Ann. Probab., 47(1), 61-108.

[27] Simon, B. and Griffiths, R. B. (1973). The $\left(\phi^{4}\right)_{2}$ field theory as a classical Ising model. Comm. Math. Phys., 33(2), 145-164.

[28] Stein, C. (1972). A bound for the error in the normal approximation to the distribution of a sum of dependent random variables. In Proceedings of the Sixth Berkeley Symposium on Mathematical Statistics and Probability, 2, pp. 583-602. University of California Press, Berkeley.

[29] Stein, C. (1986). Approximate Computation of Expectations. Institute of Mathematical Statistics Lecture Notes-Monograph Series, 7. Institute of Mathematical Statistics, Hayward, CA.

imsart-generic ver. 2020/08/06 file: AAP1589-final.tex date: April 28, 2021 